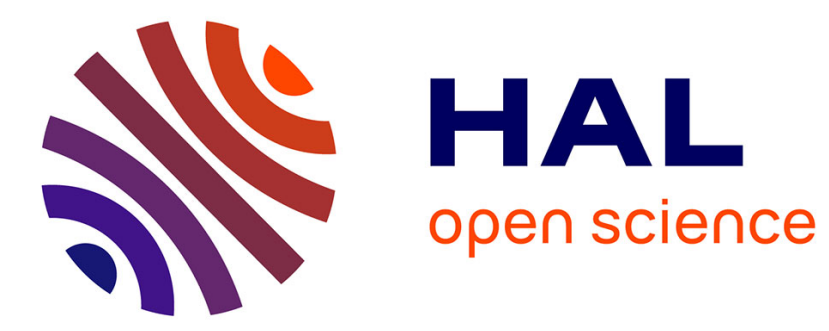

\title{
Sequential High-Resolution Direction Finding From Higher Order Statistics
}

\author{
Gwénaël Birot, Laurent Albera, Pascal Chevalier
}

\section{To cite this version:}

Gwénaël Birot, Laurent Albera, Pascal Chevalier. Sequential High-Resolution Direction Finding From Higher Order Statistics. IEEE Transactions on Signal Processing, 2010, 58 (8), pp.4144-4155. 10.1109/TSP.2010.2049569 . hal-00593309

\section{HAL Id: hal-00593309 \\ https://hal.science/hal-00593309}

Submitted on 13 May 2011

HAL is a multi-disciplinary open access archive for the deposit and dissemination of scientific research documents, whether they are published or not. The documents may come from teaching and research institutions in France or abroad, or from public or private research centers.
L'archive ouverte pluridisciplinaire HAL, est destinée au dépôt et à la diffusion de documents scientifiques de niveau recherche, publiés ou non, émanant des établissements d'enseignement et de recherche français ou étrangers, des laboratoires publics ou privés. 


\title{
Sequential High Resolution Direction Finding from Higher Order Statistics
}

\author{
Gwenael Birot, Laurent Albera, Member, IEEE, Pascal Chevalier
}

\begin{abstract}
The classical higher order MUSIC-like methods based on a simultaneous search for all Directions Of Arrival (DOA's) show i) a capacity for processing underdetermined mixtures of sources, ii) a high robustness with respect to both a Gaussian noise with unknown spatial coherence and modeling errors, and iii) a better resolution than algorithms based on second order statistics. However, these methods have some limits: for a finite number of samples, they show poor performance for sources exhibiting quasi-collinear DOA's. In order to overcome this drawback, two new sequential MUSIC-like algorithms are proposed in this paper, namely the $2 q$-D-MUSIC and the $2 q$-RAP-MUSIC $(q \geq 2)$ algorithms. These methods are based on a sequential optimization of proposed generalized noise and signal $2 q$-MUSIC metrics, respectively. That allows us to learn and then to take into account the level of correlation between sources. A comparative study, both in terms of performance and numerical complexity, is performed showing the interest of the proposed techniques when some sources are angularly close. Eventually, an upper bound of the maximum number of sources which can be processed by the $2 q$-MUSIC-like techniques is given for all $q$. This improves recent work on the $2 q$-th order virtual arrays.
\end{abstract}

Index Terms-Higher order statistics, high resolution direction finding, sequential $2 q$-MUSIC-like techniques, virtual arrays

\section{INTRODUCTION}

Many physical measurements can be modeled as a function of parameters, of which estimation is useful for solving several real-world problems. For instance, in human electrophysiology, the scalp ElectroEncephaloGraphic (EEG) data explicitly depends on the localization of brain electrical activities, which is needed in order

Copyright (c) 2010 IEEE. Personal use of this material is permitted. However, permission to use this material for any other purposes must be obtained from the IEEE by sending a request to pubspermissions@ieee.org.

This work was supported by the French Government, in one part by the ANR DECOTES contract (06-BLAN-0074) and in second part by the Brittany region contract ACOMB-SOLARE.

G. Birot and L. Albera are with the INSERM U642, Rennes F-35000, France; and the Université de Rennes 1, LTSI, Rennes F-35000, France. P. Chevalier is with THALES Communications, EDS/SPM, 146 Bvd de Valmy, BP 82, Colombes, F-92704, France. to allow for the study of neuronal dysfunctions specific to certain brain pathologies [1], [2]. Such inverse problems also appear in seismology [3], and in radiocommunications [4] when the Directions Of Arrival (DOA's) of multiple radiating sources impinging on a passive sensor array have to be estimated. Numerous algorithms were proposed to solve these estimation problems [4]-[9]. In particular, Higher Order (HO) subspace-based estimation techniques [10]-[14] such as the $2 q$-th order $(q \geq 2)$ MUSIC method [13], [14], namely 2q-MUSIC, showed i) a capacity for processing underdetermined mixtures of sources, ii) a robustness with respect to both a Gaussian noise with unknown spatial coherence and modeling errors, and iii) a better resolution, in comparison with the classical Second Order (SO) MUSIC technique [6], also called 2-MUSIC in the following.

However, in spite of the use of HO statistics, if the data collection time and/or Signal-to-Noise Ratio (SNR) are not large enough, the $2 q$-MUSIC method shows poor performance for sources exhibiting quasi-collinear DOA's. Sequential (or deflation) methods, based on an alternating projection scheme [15] and SO statistics, were proposed in order to solve these problems. This has given rise to three remarkable algorithms: S-MUSIC (Sequential MUSIC) [16], RAP-MUSIC (Recursively Applied and Projected MUSIC) [17] also called 2-RAPMUSIC in the following and IES-MUSIC (ImprovEd Sequential MUSIC) [18]. By estimating the DOA's sequentially rather than simultaneously, these three SO MUSIC-like methods remove the spatial interferences among sources and improve the resolution. Nevertheless, as for all SO MUSIC-like algorithms, the aforementioned methods cannot process underdetermined mixtures of sources, and are weakly robust with respect to both modeling errors [19], [20] and the presence of a strong background noise of unknown spatial coherence [8].

In order to overcome these drawbacks, the present paper proposes two new sequential algorithms, called $2 q$ D-MUSIC and $2 q$-RAP-MUSIC ( $q \geq 2)$. These methods are based on a sequential optimization of proposed generalized noise and signal $2 q$-MUSIC metrics, respectively. The use of the generalized metrics rather than the 
classical metrics allows us to learn and then to take into account the level of correlation between sources. Moreover, the computational load of the sequential scheme is reduced by the use of a recursively built deflation projector. The problem formulation and the $2 q$-th order statistics are given in section II. Section III introduces both new sequential $2 q$-D-MUSIC and $2 q$-RAP-MUSIC $(q \geq 2)$ techniques. Section IV presents a recursive way to compute the projectors used by the proposed methods. It also summarizes the algorithms step by step in order to facilitate their implementation and gives their numerical complexity. The computer results and the conclusion are given in sections $\mathrm{V}$ and VI, respectively.

\section{Problem Formulation AND STATISTICS}

\section{A. Problem Formulation}

Let $\{\boldsymbol{x}(t)\}$ be the vector process of the complex envelopes of the signals at the output of an array of $N$ narrow-band identical sensors, given by:

$$
\boldsymbol{x}(t)=\sum_{p=1}^{P} s_{p}(t) \boldsymbol{a}\left(\boldsymbol{\theta}_{p}\right)+\boldsymbol{\nu}(t)=\boldsymbol{A}(\Theta) \boldsymbol{s}(t)+\boldsymbol{\nu}(t)
$$

where $\boldsymbol{s}(t)=\left[s_{1}(t), \ldots, s_{P}(t)\right]^{\top}$ denotes the $P$-dimensional source vector, $\boldsymbol{A}(\Theta)=\left[\boldsymbol{a}\left(\boldsymbol{\theta}_{1}\right), \ldots, \boldsymbol{a}\left(\boldsymbol{\theta}_{P}\right)\right]$ is the $(N \times P)$ mixing matrix of the source steering vectors $\boldsymbol{a}\left(\boldsymbol{\theta}_{p}\right)$ with $\Theta=\left\{\boldsymbol{\theta}_{p}, 1 \leq p \leq P\right\}$. The vector parameter $\boldsymbol{\theta}_{p}=\left(\theta_{p}, \varphi_{p}\right)$ is the DOA of the $p$-th source where $\theta_{p}$ and $\varphi_{p}$ are the azimuth and elevation angles, respectively. The $N$-dimensional vector $\boldsymbol{\nu}(t)$ is a zero-mean Gaussian noise. Although in practice the source and noise vectors are unknown, they can be assumed to be statistically independent. Some sources can be mutually statistically dependent, but not totally coherent. As a consequence $\Theta$ can be partitioned in a set whose elements $\Theta_{g}(1 \leq$ $g \leq G)$ are subsets of $\Theta$ such that the $P_{g}$ DOA's in $\Theta_{g}$ correspond to dependent sources, while DOA's belonging to different subsets correspond to independent sources. Under these considerations, the observation vector $\boldsymbol{x}(t)$ can be rewritten as:

$$
\boldsymbol{x}(t)=\sum_{g=1}^{G} \boldsymbol{A}\left(\Theta_{g}\right) \boldsymbol{s}_{g}(t)+\boldsymbol{\nu}(t)
$$

where $\boldsymbol{s}_{g}(t)=\left[s_{g}^{1}(t), \ldots, s_{g}^{P_{g}}(t)\right]^{\top}$ denotes the $P_{g^{-}}$ dimensional vector of the statistically dependent sources whose DOA's belong to $\Theta_{g}$. The direction finding problem consists in identifying the set $\Theta$ of the $P$ source DOA's $\boldsymbol{\theta}_{p}$. In practice, the structure of $\boldsymbol{a}(\boldsymbol{\theta})$ as a function of the free parameter $\boldsymbol{\theta}$ is well-known [14].

\section{B. Data statistics}

The stochastic methods considered in this paper use the information contained in the $\left(N^{q} \times N^{q}\right) 2 q$-th order statistical matrix $C_{2 q, \boldsymbol{x}}^{\ell}$ of which entries $C_{n_{1}, \ldots, n_{q}, \boldsymbol{x}}^{n_{q+1}, \ldots, n_{2 q}}$ are the temporal mean of the $2 q$-th order cumulants [21][23] of the vector process $\{\boldsymbol{x}(t)\}$. A complete definition of this matrix is given in [14] and is not repeated here. The index $\ell(\ell \in\{0, \ldots, 2 q\})$ of the $2 q$-th order statistical matrix defines the way the statistics $C_{n_{1}, \ldots, n_{q}, \boldsymbol{x}}^{n_{q+1}, \ldots, n_{2 q}}$ are arranged [24]. It determines both the resolution and the maximal processing power of the $2 q$-th order statistical method. The optimal value of $\ell$ is the integer part of $q / 2$ [24]. Under the assumptions of section II-A, for a given value of $\ell$ and using the multilinearity property enjoyed by cumulants [21], [22], the entries of $C_{2 q, \boldsymbol{x}}^{\ell}$ have the following decomposition for $q \geq 2$ :

$$
\begin{aligned}
C_{n_{1}, \ldots, n_{q}, \boldsymbol{x}}^{n_{q+1}, \ldots, n_{2 q}}= & \sum_{g=1}^{G} \sum_{p_{1}, \ldots, p_{2 q}} C_{p_{1}, \ldots, p_{q}, \boldsymbol{s}_{g}}^{p_{q+1}, \ldots, p_{2 q}} A\left(\Theta_{g}\right)_{n_{1}, p_{1}} \ldots \\
& A\left(\Theta_{g}\right)_{n_{q}, p_{q}} A\left(\Theta_{g}\right)_{n_{q+1}, p_{q+1}}^{*} \ldots A\left(\Theta_{g}\right)_{n_{2 q}, p_{2 q}}^{*}(3)
\end{aligned}
$$

where $C_{p_{1}, \ldots, p_{q}, \boldsymbol{s}_{g}}^{p_{q+1}, \ldots, p_{2}}$ is an entry of the $\left(P_{g}^{q} \times P_{g}^{q}\right) 2 q$ th order statistical matrix $C_{2 q, s_{g}}^{\ell}$ of $\left\{s_{g}(t)\right\}$, say the temporal mean of a $2 q$-th order cumulant of the vector process $\left\{s_{g}(t)\right\}$. Component $A\left(\Theta_{g}\right)_{n, p}$ denotes the $(n, p)$-th entry of matrix $\boldsymbol{A}\left(\Theta_{g}\right)$. As a consequence, the $\left(N^{q} \times N^{q}\right) 2 q$-th order statistical matrix, $C_{2 q, \boldsymbol{x}}^{\ell}$, of $\{\boldsymbol{x}(t)\}$ has the following algebraic structure for $q \geq 2$ [14]:

$$
\boldsymbol{C}_{2 q, \boldsymbol{x}}^{\ell}=\sum_{g=1}^{G} \boldsymbol{A}_{2 q}^{\ell}\left(\Theta_{g}\right) \boldsymbol{C}_{2 q, \boldsymbol{s}_{g}}^{\ell} \boldsymbol{A}_{2 q}^{\ell}\left(\Theta_{g}\right)^{\mathrm{H}}
$$

where $\boldsymbol{A}_{2 q}^{\ell}\left(\Theta_{g}\right)=\boldsymbol{A}\left(\Theta_{g}\right)^{\otimes(q-\ell)} \otimes \boldsymbol{A}\left(\Theta_{g}\right)^{* \otimes \ell}, \otimes$ is the Kronecker product operator, $\boldsymbol{A}^{\otimes \ell}=\boldsymbol{A} \otimes \boldsymbol{A} \otimes \ldots \otimes \boldsymbol{A}$ uses the Kronecker product $\ell-1$ times. In practice, statistical matrices cannot be exactly computed and have to be estimated from one $K$-length realization of the process $\{\boldsymbol{x}(t)\}$. Unbiased and consistent estimators exist even in the case of cyclostationary data [25], [26].

\section{The $2 q$-D-MUSIC AND $2 q$-RAP-MUSIC ALGORITHMS}

Let's recall the assumptions needed by the $2 q$-MUSIClike $(q \geq 2)$ techniques [12]:

H1) Each $\Theta_{g}$ has not more than $N$ DOA's, i.e. $\forall g$, $P_{g}<N$

H2) Each matrix $\boldsymbol{A}\left(\Theta_{g}\right)$ is full column rank $P_{g}$;

H3) The matrix $\boldsymbol{A}_{2 q}^{\ell}(\Theta)=\left[\boldsymbol{A}_{2 q}^{\ell}\left(\Theta_{1}\right), \ldots, \boldsymbol{A}_{2 q}^{\ell}\left(\Theta_{G}\right)\right]$ is full column rank;

H4) The rank of $C_{2 q, x}^{\ell}, r_{2 q}^{\ell}$, is strictly lesser than the maximum rank $\mathcal{N}_{2 q}^{\ell}$ of $\boldsymbol{C}_{2 q, \boldsymbol{x}}^{\ell}$.

Under $\mathbf{H 2}$ ) and $\mathbf{H 3}$ ), $r_{2 q}^{\ell}$ is the sum of the ranks, $r_{2 q, g}^{\ell}$, of the $G$ statistical matrices $C_{2 q, s_{g}}^{\ell}$. Those matrices may not be full rank, thus $r_{2 q, g}^{\ell} \leq\left(P_{g}\right)^{q}$. 


\section{A. Signal and noise 2q-MUSIC metrics}

Under hypotheses H1) to H4), the 2q-MUSIC-like algorithms use the possibility of computing the $2 q$ th order signal subspace $\operatorname{Span}\left\{\boldsymbol{C}_{2 q, \boldsymbol{x}}^{\ell}\right\}$, spanned by the column vectors of $\boldsymbol{C}_{2 q, \boldsymbol{x}}^{\ell}$, and its complementary orthogonal subspace, called the $2 q$-th order noise subspace, by diagonalizing the statistical matrix $C_{2 q, \boldsymbol{x}}^{\ell}$ [14]:

$$
\boldsymbol{C}_{2 q, \boldsymbol{x}}^{\ell}=\boldsymbol{E}_{2 q, \boldsymbol{s}}^{\ell} \boldsymbol{\Lambda}_{2 q, \boldsymbol{s}}^{\ell}\left[\boldsymbol{E}_{2 q, \boldsymbol{s}}^{\ell}\right]^{\mathrm{H}}+\boldsymbol{E}_{2 q, \boldsymbol{\nu}}^{\ell} \boldsymbol{\Lambda}_{2 q, \boldsymbol{\nu}}^{\ell}\left[\boldsymbol{E}_{2 q, \boldsymbol{\nu}}^{\ell}\right]^{\mathrm{H}}
$$

where $\boldsymbol{\Lambda}_{2 q, s}^{\ell}$ is the diagonal matrix of the $r_{2 q}^{\ell}$ nonzero eigenvalues of $\boldsymbol{C}_{2 q, \boldsymbol{x}}^{\ell}$ and $\boldsymbol{E}_{2 q, s}^{\ell}$, so-called signal eigenmatrix, is the unitary matrix of the corresponding eigenvectors. $\Lambda_{2 q, \nu}^{\ell}$ is the diagonal matrix of the $N^{q}-r_{2 q}^{\ell}$ zero eigenvalues of $\boldsymbol{C}_{2 q, \boldsymbol{x}}^{\ell}$ and $\boldsymbol{E}_{2 q, \boldsymbol{\nu}}^{\ell}$, so-called the noise eigenmatrix, is the unitary matrix of the associated eigenvectors. Under $\mathbf{H 1}$ ) to $\mathbf{H 3}), \boldsymbol{E}_{2 q, \boldsymbol{s}}^{\ell}$ and $\boldsymbol{E}_{2 q, \boldsymbol{\nu}}^{\ell}$ are some basis of the $2 q$-th order signal and noise subspaces, respectively.

The $2 q$-MUSIC method makes use of the orthogonality between the $P 2 q$-th order steering vectors $\boldsymbol{a}_{2 q}^{\ell}\left(\boldsymbol{\theta}_{p}\right)=\boldsymbol{a}\left(\boldsymbol{\theta}_{p}\right)^{\otimes \ell} \otimes \boldsymbol{a}\left(\boldsymbol{\theta}_{p}\right)^{* \otimes(q-\ell)}$ and the $2 q$-th order noise subspace. The algorithm minimizes the metric $\Upsilon\left(\boldsymbol{a}_{2 q}^{\ell}(\boldsymbol{\theta}), \boldsymbol{E}_{2 q, \boldsymbol{\nu}}^{\ell}\right)$, called $2 q$-MUSIC noise metric, where:

$$
\Upsilon(\boldsymbol{a}, \boldsymbol{E})=\boldsymbol{a}^{\mathrm{H}} \boldsymbol{E}\left(\boldsymbol{E}^{\mathrm{H}} \boldsymbol{E}\right)^{-1} \boldsymbol{E}^{\mathrm{H}} \boldsymbol{a} /\|\boldsymbol{a}\|^{2}
$$

As suggested for $q=1$ in [17], the collinearity between the $P$ steering vectors $\boldsymbol{a}_{2 q}^{\ell}\left(\boldsymbol{\theta}_{p}\right)$ and the $2 q$-th order signal subspace can also be used by maximizing the $2 q$-th order signal metric $\Upsilon\left(\boldsymbol{a}_{2 q}^{\ell}(\boldsymbol{\theta}), \boldsymbol{E}_{2 q, \boldsymbol{s}}^{\ell}\right)$. We show in appendix VI-B that $\Upsilon\left(\boldsymbol{a}_{2 q}^{\ell}(\boldsymbol{\theta}), \boldsymbol{E}_{2 q, \boldsymbol{s}}^{\ell}\right)=1-\Upsilon\left(\boldsymbol{a}_{2 q}^{\ell}(\boldsymbol{\theta}), \boldsymbol{E}_{2 q, \boldsymbol{\nu}}^{\ell}\right)$, which implies that both metrics are theoretically related, and, more precisely, equivalent. However they may have some differences in practice, especially in terms of computational complexity. For instance, the $2 q$-MUSIC noise metric is more complex than the signal metric when $P$ is close to the upper bound of the number of sources that can be processed.

\section{B. Principle of the sequential optimization}

If the $2 q$-th order noise and signal subspaces are perfectly estimated, the $P$ sources are simply found as the $P$ minimizers of $\Upsilon\left(\boldsymbol{a}_{2 q}^{\ell}(\boldsymbol{\theta}), \boldsymbol{E}_{2 q, \boldsymbol{\nu}}^{\ell}\right)$ or the $P$ maximizers of $\Upsilon\left(\boldsymbol{a}_{2 q}^{\ell}(\boldsymbol{\theta}), \boldsymbol{E}_{2 q, \boldsymbol{s}}^{\ell}\right)$. However, errors in our estimate (due to an estimation of $C_{2 q, x}^{\ell}$ from a limited number of samples or to modeling errors) combined with a "close source" configuration, may reduce these two criteria to a function with less than $P$ optima. In this situation, the spatial interferences between close sources produce only one peak on the metrics (fig. 1) and therefore some sources cannot be identified. Accordingly, we propose

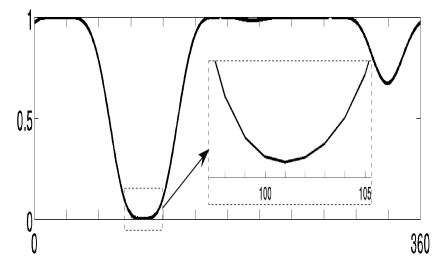

(a) noise metric



(b) signal metric
Fig. 1. Sixth order noise and signal metrics from a noisy mixture of two QPSK sources of length 10000 impinging on a UCA of four sensors with DOA's given by $\boldsymbol{\theta}_{1}=[0,100]^{\top}$ and $\boldsymbol{\theta}_{2}=[0,102.5]$, respectively, and a SNR of $15 \mathrm{~dB}$. Only one peak appears in spite of the presence of two sources.

to sequentially process the $P$ sources rather than simultaneously: the $(p+1)$-th source DOA is identified by optimizing a metric built from a new signal subspace, the contribution of the already estimated $p$ source DOA's having been removed. This procedure allows us to cancel the spatial interferences between close sources and to identify the $P$ sources even if less than $P$ peaks appear in the initial metrics. Let $\boldsymbol{P}_{2 q}^{\ell}\left(\Theta^{p}\right)$ be an orthogonal deflation projector able to remove from the $2 q$-th order signal subspace all contributions of the DOA's in $\Theta^{p}=$ $\left\{\boldsymbol{\theta}_{1}, \ldots, \boldsymbol{\theta}_{p}\right\}$. The application of $\boldsymbol{P}_{2 q}^{\ell}\left(\Theta^{p}\right)$ to matrices $\boldsymbol{C}_{2 q, \boldsymbol{x}}^{\ell}$ and $\boldsymbol{E}_{2 q, \boldsymbol{s}}^{\ell}$ leads to the $2 q$-D-MUSIC and $2 q$ RAP-MUSIC algorithms, respectively. Having $\Theta^{p}, 2 q-$ D-MUSIC identifies the $(p+1)$-th DOA as following:

$$
\hat{\boldsymbol{\theta}}_{p+1}=\arg \min _{\boldsymbol{\theta}} \Upsilon\left(\boldsymbol{P}_{2 q}^{\ell}\left(\Theta^{p}\right) \boldsymbol{a}_{2 q}^{\ell}(\boldsymbol{\theta}), \boldsymbol{E}_{2 q, \boldsymbol{\nu}}^{\ell, p}\right)
$$

where $\boldsymbol{E}_{2 q, \boldsymbol{\nu}}^{\ell, p}$ is the noise eigenmatrix computed from the deflated statistical matrix $\boldsymbol{P}_{2 q}^{\ell}\left(\Theta^{p}\right) \boldsymbol{C}_{2 q, \boldsymbol{x}}^{\ell} \boldsymbol{P}_{2 q}^{\ell}\left(\Theta^{p}\right)$. The $2 q$-RAP-MUSIC algorithm estimates $\boldsymbol{\theta}_{p+1}$ by:

$$
\hat{\boldsymbol{\theta}}_{p+1}=\arg \max _{\boldsymbol{\theta}} \Upsilon\left(\boldsymbol{P}_{2 q}^{\ell}\left(\Theta^{p}\right) \boldsymbol{a}_{2 q}^{\ell}(\boldsymbol{\theta}), \boldsymbol{P}_{2 q}^{\ell}\left(\Theta^{p}\right) \boldsymbol{E}_{2 q, \boldsymbol{s}}^{\ell}\right)
$$

Both sequential methods are initialized by $\Theta^{0}=\varnothing$ and $\boldsymbol{P}_{2 q}^{\ell}(\varnothing)=\mathbf{I}_{N^{q}}$. They end when $\operatorname{Span}\left\{\boldsymbol{E}_{2 q, \nu}^{\ell, p}\right\}=\mathbb{C}^{N^{q}}$ and $\operatorname{Span}\left\{\boldsymbol{P}_{2 q}^{\ell}\left(\Theta^{p}\right) \boldsymbol{E}_{2 q, \boldsymbol{s}}^{\ell}\right\}=\mathbf{0}$, respectively, where $\mathbf{0}$ is the zero vector of $\mathbb{C}^{N^{q}}$.

\section{Toward an optimal orthogonal deflation projector}

The main core of the proposed algorithms is the computation of the appropriate orthogonal projector $\boldsymbol{P}_{2 q}^{\ell}\left(\Theta^{p}\right)$. From results in matrix analysis [27], such an orthogonal projector is given by:

$$
\boldsymbol{P}_{2 q}^{\ell}\left(\Theta^{p}\right)=\mathbf{I}_{N^{q}}-\boldsymbol{B}_{2 q}^{\ell}\left(\Theta^{p}\right)\left(\boldsymbol{B}_{2 q}^{\ell}\left(\Theta^{p}\right)^{\mathrm{H}} \boldsymbol{B}_{2 q}^{\ell}\left(\Theta^{p}\right)\right)^{-1} \boldsymbol{B}_{2 q}^{\ell}\left(\Theta^{p}\right)^{\mathrm{H}}
$$

where $\boldsymbol{B}_{2 q}^{\ell}\left(\Theta^{p}\right)$, so-called the null-space matrix, is full column rank with $\operatorname{Span}\left\{\boldsymbol{B}_{2 q}^{\ell}\left(\Theta^{p}\right)\right\}$ the null-space of the projection, i.e. for every vector $\boldsymbol{y}$ belonging to $\operatorname{Span}\left\{\boldsymbol{B}_{2 q}^{\ell}\left(\Theta^{p}\right)\right\}$ we have $\boldsymbol{P}_{2 q}^{\ell}\left(\Theta^{p}\right) \boldsymbol{y}=\mathbf{0}$. 
For our purposes, the appropriate null-space of the projection should be the subspace spanned by all the vectors of the $2 q$-th order signal subspace involving one or several DOA's in $\Theta^{p}$. As a result, the column vectors of the appropriate matrix $\boldsymbol{B}_{2 q}^{\ell}\left(\Theta^{p}\right)$ are necessarily linked to the column vectors of $\boldsymbol{A}_{2 q}^{\ell}(\Theta)$ involving DOA's in $\Theta^{p}$. If all sources are statistically independent, say, $G=P$, the null-space matrix is simply given by $\boldsymbol{B}_{2 q}^{\ell}\left(\Theta^{p}\right)=\left[\boldsymbol{a}_{2 q}^{\ell}\left(\boldsymbol{\theta}_{1}\right), \ldots, \boldsymbol{a}_{2 q}^{\ell}\left(\boldsymbol{\theta}_{p}\right)\right]$. Nevertheless, if some sources are correlated, say $G<P$, then the computation of matrix $\boldsymbol{B}_{2 q}^{\ell}\left(\Theta^{p}\right)$ is not so trivial since some vectors in $\operatorname{Span}\left\{\boldsymbol{B}_{2 q}^{\ell}\left(\Theta^{p}\right)\right\}$ may also involve DOA's that have not been estimated at step $p$. For instance, for $G=1$ and $q=$ 2 , at the second step of the Fourth Order (FO) sequential procedure, the $2(P-1)$ cross vectors $\boldsymbol{a}\left(\boldsymbol{\theta}_{1}\right) \otimes \boldsymbol{a}\left(\boldsymbol{\theta}_{2}\right)^{*}, \ldots$, $\boldsymbol{a}\left(\boldsymbol{\theta}_{1}\right) \otimes \boldsymbol{a}\left(\boldsymbol{\theta}_{P}\right)^{*}$ and $\boldsymbol{a}\left(\boldsymbol{\theta}_{2}\right) \otimes \boldsymbol{a}\left(\boldsymbol{\theta}_{1}\right)^{*}, \ldots, \boldsymbol{a}\left(\boldsymbol{\theta}_{P}\right) \otimes \boldsymbol{a}\left(\boldsymbol{\theta}_{1}\right)^{*}$ must belong to the null-space $\operatorname{Span}\left\{\boldsymbol{B}_{4}^{1}\left(\Theta^{1}\right)\right\}$ of the projection but the corresponding matrix $\boldsymbol{B}_{4}^{1}\left(\Theta^{1}\right)$ cannot be built since DOA's $\boldsymbol{\theta}_{2}, \ldots, \boldsymbol{\theta}_{P}$ remain unknown at this step of the FO sequential scheme. One solution is to build a null-space, in which $\operatorname{Span}\left\{\boldsymbol{B}_{2 q}^{\ell}\left(\Theta^{p}\right)\right\}$ is included but not $\operatorname{Span}\left\{\left[\boldsymbol{a}_{2 q}^{\ell}\left(\boldsymbol{\theta}_{p+1}\right), \ldots, \boldsymbol{a}_{2 q}^{\ell}\left(\boldsymbol{\theta}_{P}\right)\right]\right\}$. The orthogonal projector associated to this null-space can be computed as:

$$
\boldsymbol{P}_{2 q}^{\ell}\left(\Theta^{p}\right)=\left(\boldsymbol{P}_{2}^{1}\left(\Theta^{p}\right)\right)^{\otimes(\ell)} \otimes\left(\boldsymbol{P}_{2}^{1}\left(\Theta^{p}\right)\right)^{* \otimes(q-\ell)}
$$

where:

$$
\boldsymbol{P}_{2}^{1}\left(\Theta^{p}\right)=\mathbf{I}_{N}-\boldsymbol{A}\left(\Theta^{p}\right)\left(\boldsymbol{A}\left(\Theta^{p}\right)^{\mathrm{H}} \boldsymbol{A}\left(\Theta^{p}\right)\right)^{-1} \boldsymbol{A}\left(\Theta^{p}\right)^{\mathrm{H}}
$$

The proof is given in appendix VI-A. However, since (10) is computed from the SO projector $\boldsymbol{P}_{2}^{\ell}\left(\Theta^{p}\right)$, it is not defined if $\boldsymbol{B}_{2}^{\ell}\left(\Theta^{p}\right)^{\mathrm{H}} \boldsymbol{B}_{2}^{\ell}\left(\Theta^{p}\right)=\boldsymbol{A}\left(\Theta^{p}\right)^{\mathrm{H}} \boldsymbol{A}\left(\Theta^{p}\right)$ is not invertible, say if $p>N$.

A way of building a more optimal projector at order $2 q$ consists of trying to identify all the column vectors of the null-space matrix $\boldsymbol{B}_{2 q}^{\ell}\left(\Theta^{p}\right)$, even if some of them involve DOA's yet to be estimated. Thus, we will be able to build projector (9). Let's consider first the particular case where $(q, \ell)=(2,2), P=2$ and $G=1$. The FO virtual mixing matrix $\boldsymbol{A}_{4}^{2}(\Theta)$ is given by:

$\boldsymbol{A}_{4}^{2}(\Theta)=\left[\boldsymbol{a}_{4}^{2}\left(\boldsymbol{\theta}_{1}\right), \boldsymbol{a}\left(\boldsymbol{\theta}_{1}\right) \otimes \boldsymbol{a}\left(\boldsymbol{\theta}_{2}\right), \boldsymbol{a}\left(\boldsymbol{\theta}_{2}\right) \otimes \boldsymbol{a}\left(\boldsymbol{\theta}_{1}\right), \boldsymbol{a}_{4}^{2}\left(\boldsymbol{\theta}_{2}\right)\right]$

and the 3-dimensional signal subspace is spanned by $\left\{\boldsymbol{a}_{4}^{2}\left(\boldsymbol{\theta}_{1}\right), \boldsymbol{a}\left(\boldsymbol{\theta}_{1}\right) \otimes \boldsymbol{a}\left(\boldsymbol{\theta}_{2}\right)+\boldsymbol{a}\left(\boldsymbol{\theta}_{2}\right) \otimes \boldsymbol{a}\left(\boldsymbol{\theta}_{1}\right), \boldsymbol{a}_{4}^{2}\left(\boldsymbol{\theta}_{2}\right)\right\}$. In this case, the column vectors of the FO virtual mixing matrix do not form a basis of the FO signal subspace. Indeed, the statistical matrix $C_{4, \boldsymbol{x}}^{2}$ of the obervations is given by $\boldsymbol{A}_{4}^{2}(\Theta) \boldsymbol{C}_{4, \boldsymbol{s}}^{2} \boldsymbol{A}_{4}^{2}(\Theta)^{\mathrm{H}}$. Using the fact that, for all $1 \leq p_{1}, p_{2}, p_{3}, p_{4} \leq P, C_{p_{1}, p_{2}, s}^{p_{3}, p_{4}}=C_{p_{1}, p_{2}, s}^{p_{4}, p_{3}}=C_{p_{2}, p_{1}, s}^{p_{3}, p_{4}}=$ $C_{p_{2}, p_{1}, s}^{p_{4}, p_{3}}$, we can remark that the second and third rows of $C_{4, s}^{2}$ are equal, and that its second and third columns are also equal. As a matter of fact, $C_{4, x}^{2}$ can be factorized as $\tilde{\boldsymbol{A}}_{4}^{2}(\Theta) \tilde{\boldsymbol{C}}_{4, \boldsymbol{s}}^{2} \tilde{\boldsymbol{A}}_{4}^{2}(\Theta)^{\mathrm{H}}$ where $\tilde{\boldsymbol{A}}_{4}^{2}=\left[\boldsymbol{a}\left(\boldsymbol{\theta}_{1}\right) \otimes \boldsymbol{a}\left(\boldsymbol{\theta}_{1}\right), \boldsymbol{a}\left(\boldsymbol{\theta}_{1}\right) \otimes\right.$ $\left.\boldsymbol{a}\left(\boldsymbol{\theta}_{2}\right)+\boldsymbol{a}\left(\boldsymbol{\theta}_{2}\right) \otimes \boldsymbol{a}\left(\boldsymbol{\theta}_{1}\right), \boldsymbol{a}\left(\boldsymbol{\theta}_{2}\right) \otimes \boldsymbol{a}\left(\boldsymbol{\theta}_{2}\right)\right]$ is called the reduced mixing matrix. The matrix $\tilde{C}_{4, s}^{2}$, assumed to be full rank and called the reduced statistical matrix of the sources, is the concatenation of the different rows and columns of $C_{4, s}^{2}$. As a result, for $p=1$, the appropriate matrix $\boldsymbol{B}_{4}^{2}\left(\Theta^{1}\right)$ is $\left[\boldsymbol{a}_{4}^{2}\left(\boldsymbol{\theta}_{1}\right), \boldsymbol{a}\left(\boldsymbol{\theta}_{1}\right) \otimes \boldsymbol{a}\left(\boldsymbol{\theta}_{2}\right)+\boldsymbol{a}\left(\boldsymbol{\theta}_{2}\right) \otimes \boldsymbol{a}\left(\boldsymbol{\theta}_{1}\right)\right]$ while the null-space matrix of the suboptimal projector (10) is $\left[\boldsymbol{a}_{4}^{2}\left(\boldsymbol{\theta}_{1}\right), \boldsymbol{a}\left(\boldsymbol{\theta}_{1}\right) \otimes \boldsymbol{a}\left(\boldsymbol{\theta}_{2}\right), \boldsymbol{a}\left(\boldsymbol{\theta}_{2}\right) \otimes \boldsymbol{a}\left(\boldsymbol{\theta}_{1}\right)\right]$.

More generally, we can show that $\boldsymbol{B}_{2 q}^{\ell}\left(\Theta^{p}\right)$ is composed of column vectors of the reduced $2 q$-th order virtual mixing matrix $\tilde{\boldsymbol{A}}_{2 q}^{\ell}(\Theta)=\left[\tilde{\boldsymbol{A}}_{2 q}^{\ell}\left(\Theta_{1}\right), \ldots, \tilde{\boldsymbol{A}}_{2 q}^{\ell}\left(\Theta_{G}\right)\right]$. More precisely, it should be defined as the concatenation of the column vectors of matrices $\tilde{\boldsymbol{A}}_{2 q}^{\ell}\left(\Theta_{g}\right)$ involving DOA's in $\Theta^{p}$ :

$$
\boldsymbol{B}_{2 q}^{\ell}\left(\Theta^{p}\right)=\left[\tilde{\boldsymbol{A}}_{2 q}^{\ell}\left(\Theta_{1}^{p}\right), \tilde{\boldsymbol{A}}_{2 q}^{\ell}\left(\Theta_{2}^{p}\right), \ldots, \tilde{\boldsymbol{A}}_{2 q}^{\ell}\left(\Theta_{G}^{p}\right)\right]
$$

where $\Theta_{g}^{p}=\Theta^{p} \cap \Theta_{g}$ is the set containing the DOA's of $\Theta^{p}$ belonging to $\Theta_{g}$ and where $\tilde{\boldsymbol{A}}_{2 q}^{\ell}\left(\Theta_{g}^{p}\right)$ contains the column vectors of $\tilde{\boldsymbol{A}}_{2 q}^{\ell}\left(\Theta_{g}\right)$ involving DOA's in $\Theta_{g}^{p}$. In order to explicitly formulate the column vectors of $\tilde{\boldsymbol{A}}_{2 q}^{\ell}\left(\Theta_{g}^{p}\right)$, we give a definition of the generalized $2 q$-th order steering vector:

$$
\boldsymbol{a}_{2 q}^{\ell}\left(\boldsymbol{\theta}_{p_{1}}, \ldots, \boldsymbol{\theta}_{p_{q}}\right)=\bigotimes_{i=1}^{\ell} \boldsymbol{a}\left(\boldsymbol{\theta}_{p_{i}}\right) \bigotimes_{j=\ell+1}^{q} \boldsymbol{a}\left(\boldsymbol{\theta}_{p_{j}}\right)^{*}
$$

where $\bigotimes_{i=1}^{q} \boldsymbol{a}_{i}=\boldsymbol{a}_{1} \otimes \boldsymbol{a}_{2} \otimes \ldots \otimes \boldsymbol{a}_{q}$. The $\left(N^{q} \times\left(P_{g}\right)^{q}\right)$ matrix $\boldsymbol{A}_{2 q}^{\ell}\left(\Theta_{g}\right)$ is the horizontal concatenation of the $\left(P_{g}\right)^{q}$ column vectors $\boldsymbol{a}_{2 q}^{\ell}\left(\boldsymbol{\theta}_{p_{1}}, \ldots, \boldsymbol{\theta}_{p_{q}}\right), \boldsymbol{\theta}_{p_{i}} \in \Theta_{g}, 1 \leq p_{i} \leq P_{g}$. Due to the redundant rows and columns of the statistical matrix $C_{2 q, s_{g}}^{\ell}$, we can show that:

$$
\boldsymbol{A}_{2 q}^{\ell}\left(\Theta_{g}\right) \boldsymbol{C}_{2 q, \boldsymbol{s}_{g}}^{\ell} \boldsymbol{A}_{2 q}^{\ell}\left(\Theta_{g}\right)^{\mathrm{H}}=\tilde{\boldsymbol{A}}_{2 q}^{\ell}\left(\Theta_{g}\right) \tilde{\boldsymbol{C}}_{2 q, \boldsymbol{s}_{g}}^{\ell} \tilde{\boldsymbol{A}}_{2 q}^{\ell}\left(\Theta_{g}\right)^{\mathrm{H}}
$$

where $\tilde{\boldsymbol{C}}_{2 q, \boldsymbol{s}_{g}}^{\ell}$ is the $\left(r_{2 q, g}^{\ell} \times r_{2 q, g}^{\ell}\right)$ reduced statistical matrix of $\left\{\boldsymbol{s}_{g}(t)\right\}$. This matrix is the concatenation of all the non-redundant rows and columns of $C_{2 q, s_{g}}^{\ell}$. Thus under $\mathbf{H 3}$ ) and assuming that $\tilde{\boldsymbol{C}}_{2 q, \boldsymbol{s}_{g}}^{\ell}$ is full rank, the $r_{2 q, g}^{\ell}$ linearly independent column vectors of $\tilde{\boldsymbol{A}}_{2 q}^{\ell}\left(\Theta_{g}\right)$, $\tilde{\boldsymbol{a}}_{2 q, g}^{\ell}\left(\boldsymbol{\theta}_{p_{1}}, \ldots, \boldsymbol{\theta}_{p_{q}}\right)$, for which $1 \leq p_{1} \leq \ldots \leq p_{\ell} \leq P_{g}$ and $1 \leq p_{\ell+1} \leq \ldots \leq p_{q} \leq P_{g}$ form a basis of $\operatorname{Span}\left\{\boldsymbol{A}_{2 q}^{\ell}\left(\Theta_{g}\right) \boldsymbol{C}_{2 q, \boldsymbol{s}_{g}}^{\ell} \boldsymbol{A}_{2 q}^{\ell}\left(\Theta_{g}\right)^{\mathrm{H}}\right\}$, where:

$$
\begin{aligned}
& \tilde{\boldsymbol{a}}_{2 q, g}^{\ell}\left(\boldsymbol{\theta}_{p_{1}}, \ldots, \boldsymbol{\theta}_{p_{q}}\right)= \\
& \sum_{k_{1}, k_{2}} \boldsymbol{a}_{2 q, g}^{\ell}\left(\sigma_{k_{1}}\left(\boldsymbol{\theta}_{p_{1}}, \ldots, \boldsymbol{\theta}_{p_{\ell}}\right), \sigma_{k_{2}}\left(\boldsymbol{\theta}_{p_{\ell+1}}, \ldots, \boldsymbol{\theta}_{p_{q}}\right)\right)
\end{aligned}
$$

Note that $\sigma_{i}\left(\boldsymbol{\theta}_{p_{1}}, \ldots, \boldsymbol{\theta}_{p_{q}}\right)$ is the $i$-th distinguishable permutation of $\left\{\boldsymbol{\theta}_{p_{1}}, \ldots, \boldsymbol{\theta}_{p_{q}}\right\}$. Matrix $\tilde{\boldsymbol{A}}_{2 q}^{\ell}\left(\Theta_{g}^{p}\right)$ is the 
concatenation of the column vectors $\tilde{\boldsymbol{a}}_{2 q, g}^{\ell}\left(\boldsymbol{\theta}_{p_{1}}, \ldots, \boldsymbol{\theta}_{p_{q}}\right)$ involving DOA's in $\Theta_{g}^{p}$. For instance, the column vectors of $\tilde{\boldsymbol{A}}_{4}^{\ell}\left(\Theta_{g}\right)$ are given in table I for $P_{g}=2$ :

\begin{tabular}{|c|c|c|}
\hline$\ell$ & $p_{1}, p_{2}$ & $\tilde{\boldsymbol{a}}_{4, g}^{\ell}\left(\boldsymbol{\theta}_{p_{1}}, \boldsymbol{\theta}_{p_{2}}\right)$ \\
\hline & 1,1 & $\boldsymbol{a}\left(\theta_{1}\right) \otimes \boldsymbol{a}\left(\theta_{1}\right)^{*}$ \\
1 & 1,2 & $\boldsymbol{a}\left(\theta_{1}\right) \otimes \boldsymbol{a}\left(\theta_{2}\right)^{*}$ \\
& 2,1 & $\boldsymbol{a}\left(\theta_{2}\right) \otimes \boldsymbol{a}\left(\theta_{1}\right)^{*}$ \\
& 2,2 & $\boldsymbol{a}\left(\theta_{2}\right) \otimes \boldsymbol{a}\left(\theta_{2}\right)^{*}$ \\
\hline \multirow{4}{*}{2} & 1,1 & $\boldsymbol{a}\left(\theta_{1}\right) \otimes \boldsymbol{a}\left(\theta_{1}\right)$ \\
& 1,2 & $\boldsymbol{a}\left(\theta_{1}\right) \otimes \boldsymbol{a}\left(\theta_{2}\right)+\boldsymbol{a}\left(\theta_{2}\right) \otimes \boldsymbol{a}\left(\theta_{1}\right)$ \\
& 2,2 & $\boldsymbol{a}\left(\theta_{2}\right) \otimes \boldsymbol{a}\left(\theta_{2}\right)$ \\
\hline
\end{tabular}

TABLE I

VECTORS $\tilde{\boldsymbol{a}}_{4, g}^{\ell}\left(\boldsymbol{\theta}_{p_{1}}, \boldsymbol{\theta}_{p_{2}}\right)$ FOR $P_{g}=2$ AS A FUNCTION OF $p_{1}, p_{2}$ AND $\ell$.

Since we know the definition of the optimal orthogonal projector $\boldsymbol{P}_{2 q}^{\ell}\left(\Theta^{p}\right)$ through equations (9), (12) and (15), we can wonder if the latter can be computed in practical contexts. In fact, there are two main problems occurring in practice. First, the knowledge of $\Theta^{p}$ may not be sufficient to build the $G$ matrices $\tilde{A}_{2 q}^{\ell}\left(\Theta_{g}^{p}\right)$. For example, if $(q, \ell)=(2,2), P=2$ and $G=1$, the knowledge of $\Theta^{1}=\left\{\boldsymbol{\theta}_{1}\right\}$ does not allow for a computation of $\tilde{\boldsymbol{A}}_{4}^{2}\left(\Theta_{1}^{1}\right)=\left[\tilde{\boldsymbol{a}}_{4}^{2}\left(\boldsymbol{\theta}_{1}, \boldsymbol{\theta}_{1}\right), \tilde{\boldsymbol{a}}_{4}^{2}\left(\boldsymbol{\theta}_{1}, \boldsymbol{\theta}_{2}\right)\right]$ since $\boldsymbol{\theta}_{2}$ has not yet been estimated at this step of the FO sequential procedure. Second, in practical context, we do not know a priori the value of $G$. In other words, the correlation between the $P$ sources is not known $a$ priori. Thus, for the considered example, even if $\boldsymbol{\theta}_{2}$ were known, we do not know if sources 1 and 2 are correlated and consequently if $\tilde{\boldsymbol{a}}_{4}^{2}\left(\boldsymbol{\theta}_{1}, \boldsymbol{\theta}_{2}\right)$ has to be removed from the FO signal subspace. In order to overcome these difficulties, we propose a generalized $2 q$-MUSIC metric allowing for identifying the generalized HO reduced steering vectors $\tilde{\boldsymbol{a}}_{2 q}^{\ell}\left(\boldsymbol{\theta}_{p_{1}}, \ldots, \boldsymbol{\theta}_{p_{q}}\right)$ that actually belong to the HO signal subspace.

\section{Generalized 2q-MUSIC metrics and sequential opti- mization}

Since we do not know a priori the correlations between the $P$ sources, we assume that for all $1 \leq p_{1}, \ldots, p_{q} \leq P$, the column vectors $\tilde{\boldsymbol{a}}_{2 q}^{\ell}\left(\boldsymbol{\theta}_{p_{1}}, \ldots, \boldsymbol{\theta}_{p_{q}}\right)$ may be in the $2 q$-th order signal subspace. Now, considering $q$ free vector parameters $\boldsymbol{\theta}^{(i)}$ such that $\boldsymbol{\theta}(1) \leq \boldsymbol{\theta}^{(2)} \leq \ldots \leq \boldsymbol{\theta}^{(\ell)}$ and $\boldsymbol{\theta}^{(\ell+1)} \leq \boldsymbol{\theta}^{(\ell+2)} \leq \ldots \leq \boldsymbol{\theta}^{(q)}$, the metrics $\Upsilon\left(\tilde{\boldsymbol{a}}_{2 q}^{\ell}\left(\boldsymbol{\theta}^{(1)}, \ldots, \boldsymbol{\theta}^{(\bar{q})}\right), \boldsymbol{E}_{2 q, \boldsymbol{\nu}}^{\ell}\right)$ (noise) and $\Upsilon\left(\tilde{\boldsymbol{a}}_{2 q}^{\ell}\left(\boldsymbol{\theta}^{(1)}, \ldots, \boldsymbol{\theta}^{(q)}\right), \boldsymbol{E}_{2 q, \boldsymbol{s}}^{\ell}\right) \quad$ (signal), called generalized $2 q$-MUSIC metrics, are equal to zero and one, respectively, if and only if the $q$ parameters $\boldsymbol{\theta}^{(i)}$ belong to the same set $\Theta_{g}$. An example of the FO generalized signal metric is given in figure

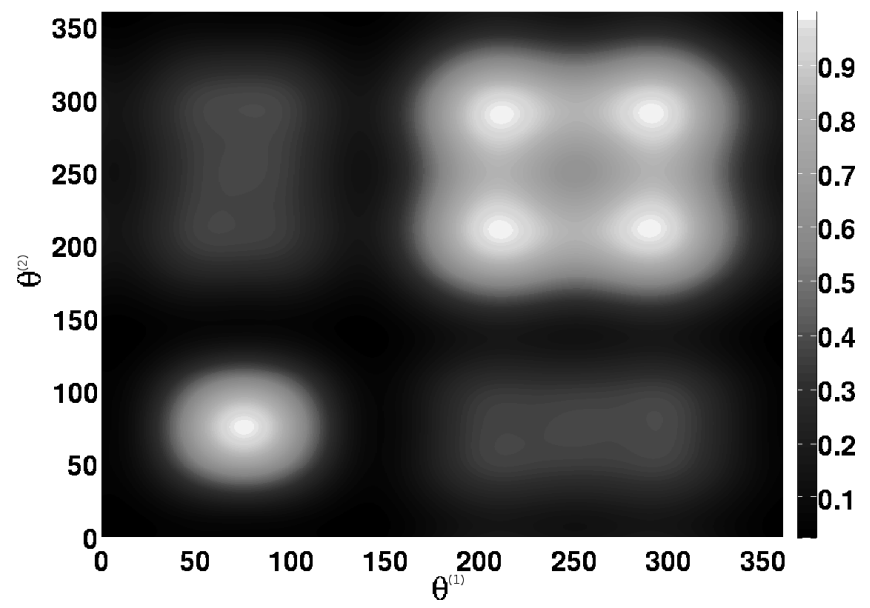

Fig. 2. Generalized FO signal metric for $(q, \ell)=(2,1), P=3$, $\Theta_{1}=\left\{80^{\circ}\right\}$ and $\Theta_{2}=\left\{210^{\circ}, 300^{\circ}\right\}$.

2, for $(q, \ell)=(2,1), P=3, \Theta_{1}=\left\{80^{\circ}\right\}$ and $\Theta_{2}=\left\{210^{\circ}, 300^{\circ}\right\}$. The three peaks in the diagonal of the metric correspond to the presence of the three FO steering vectors $\boldsymbol{a}_{4}^{1}\left(\boldsymbol{\theta}_{p}\right)=\tilde{\boldsymbol{a}}_{4}^{1}\left(\boldsymbol{\theta}_{p}, \boldsymbol{\theta}_{p}\right)$. Both remaining peaks are due to the correlation between the second and the third sources. They correspond to the presence of the vectors $\tilde{\boldsymbol{a}}_{4}^{1}\left(\boldsymbol{\theta}_{2}, \boldsymbol{\theta}_{3}\right)=\boldsymbol{a}\left(\boldsymbol{\theta}_{2}\right) \otimes \boldsymbol{a}\left(\boldsymbol{\theta}_{3}\right)^{*}$ and $\tilde{\boldsymbol{a}}_{4}^{1}\left(\boldsymbol{\theta}_{3}, \boldsymbol{\theta}_{2}\right)=\boldsymbol{a}\left(\boldsymbol{\theta}_{3}\right) \otimes \boldsymbol{a}\left(\boldsymbol{\theta}_{2}\right)^{*}$ in the FO signal subspace. As a result, the generalized $2 q$-th order noise and signal metrics can be used in order to learn whether sources are correlated. More generally, the generalized $2 q$-th order metrics allow for an identification of all steering vectors involving the $p$-th source. Consequently, it is used for the construction of an optimal orthogonal projector able to cancel the contribution of the $p$-th source from the HO signal subspace. More precisely, when $p-1$ DOA's has been estimated, we first identify the $p$-th DOA $\boldsymbol{\theta}_{p}$ using either (7) or (8). In other words, we estimate the steering vector $\boldsymbol{a}_{2 q}^{\ell}\left(\boldsymbol{\theta}_{p}\right)$. Next, its contribution is removed from the $2 q$-th order signal subspace using the orthogonal projector (9) where $\boldsymbol{B}_{2 q}^{\ell}\left(\Theta^{p}\right)=\left[\boldsymbol{B}_{2 q}^{\ell}\left(\Theta^{p-1}\right), \boldsymbol{a}_{2 q}^{\ell}\left(\boldsymbol{\theta}_{p}\right)\right]$. Then, we explore the generalized $2 q$-th order noise metric $\Upsilon\left(\boldsymbol{P}_{2 q}^{\ell}\left(\Theta^{p}\right) \tilde{\boldsymbol{a}}_{2 q}^{\ell}\left(\boldsymbol{\theta}_{p}, \ldots, \boldsymbol{\theta}_{p}, \boldsymbol{\theta}\right), \boldsymbol{E}^{\ell, p_{2 q}, \boldsymbol{\nu}}\right)$ or the equivalent generalized $2 q$-th order signal metric $\Upsilon\left(\boldsymbol{P}_{2 q}^{\ell}\left(\Theta^{p}\right) \tilde{\boldsymbol{a}}_{2 q}^{\ell}\left(\boldsymbol{\theta}_{p}, \ldots, \boldsymbol{\theta}_{p}, \boldsymbol{\theta}\right), \boldsymbol{P}_{2 q}^{\ell}\left(\Theta^{p}\right) \boldsymbol{E}_{2 q, \boldsymbol{s}}^{\ell}\right)$ in order to find a source of DOA $\boldsymbol{\theta}_{p, 1}$, potentially correlated with the $p$-th identified source. If need be, matrix $\boldsymbol{B}_{2 q}^{\ell}\left(\Theta^{p}\right)$ is updated by concatenating the previous matrix $\boldsymbol{B}_{2 q}^{\ell}\left(\Theta^{p}\right)$ and all different vectors of form $\tilde{\boldsymbol{a}}_{2 q}^{\ell}\left(\boldsymbol{\theta}^{(1)}, \ldots, \boldsymbol{\theta}^{(q)}\right)$ where the $q$ parameters $\boldsymbol{\theta}^{(i)}$ are equal to either $\boldsymbol{\theta}_{p}$ or $\boldsymbol{\theta}_{p, 1}$ but not all equal. Next, we look for a second source of DOA $\boldsymbol{\theta}_{p, 2}$, potentially correlated with the $p$-th identified source, iterating until the identification of all sources correlated with the $p$-th identified source. 
At iteration $k$ of this process, $\boldsymbol{B}_{2 q}^{\ell}\left(\Theta^{p}\right)$ is updated by concatenating the matrix $\boldsymbol{B}_{2 q}^{\ell}\left(\Theta^{p}\right)$ obtained at iteration $k-1$ and all different vectors of form $\tilde{\boldsymbol{a}}_{2 q}^{\ell}\left(\boldsymbol{\theta}^{(1)}, \ldots, \boldsymbol{\theta}^{(q)}\right)$ where at least one parameter $\boldsymbol{\theta}^{(i)}$ is equal to $\boldsymbol{\theta}_{p}$ and at least another one equal to $\boldsymbol{\theta}_{p, k}$. The other parameters $\boldsymbol{\theta}^{(i)}$ are equal to $\boldsymbol{\theta}_{p, 1}$ and/or $\boldsymbol{\theta}_{p, 2}$ and/or $\ldots$ and/or $\boldsymbol{\theta}_{p, k-1}$. Finally, we look for the $(p+1)$-th DOA using (7) or (8), and the projector $\boldsymbol{P}_{2 q}^{\ell}\left(\Theta^{p}\right)$ (9) built from the updated matrix $\boldsymbol{B}_{2 q}^{\ell}\left(\Theta^{p}\right)$.

\section{E. Identifiability of the presented techniques}

The $2 q$-MUSIC like algorithms can identify the DOA's while the rank $r_{2 q}^{\ell}$ of the $2 q$-th order signal subspace is less than the maximum rank $\mathcal{N}_{2 q}^{\ell}$ of the virtual mixing matrix $\boldsymbol{A}_{2 q}^{\ell}(\Theta)$. The theory of virtual arrays [24] gives some upper bounds of $\mathcal{N}_{2 q}^{\ell}$ in the case of independent sources but only for $1 \leq q \leq 4$. In such a case, say $G=P$, the rank $r_{2 q}^{\ell}$ is equal to $P$, thus the $2 q$-MUSIClike algorithms are theoretically able to identify up to $\mathcal{N}_{2 q}^{\ell}-1$ independent sources. In the case of correlated sources, the rank $r_{2 q}^{\ell}$ has never been discussed. Based on the computation of the $G$ reduced statistical matrices $\tilde{\boldsymbol{C}}_{2 q, s_{g}}^{\ell}$, assumed to be full rank, $r_{2 q}^{\ell}$ is deduced from the dimension of $\tilde{C}_{2 q, s_{g}}^{\ell}$ :

$$
r_{2 q}^{\ell}=\sum_{g=1}^{G} r_{2 q, g}^{\ell}=\sum_{g=1}^{G} \frac{\left(P_{g}+\ell-1\right) !}{\ell !\left(P_{g}-1\right) !} \frac{\left(P_{g}+q-\ell-1\right) !}{(q-\ell) !\left(P_{g}-1\right) !}
$$

where $r_{2 q, g}^{\ell}$ is the dimension of the square matrix $\tilde{\boldsymbol{C}}_{2 q, \boldsymbol{s}_{g}}^{\ell}$. The 2q-MUSIC-like algorithms can identify the sources if $r_{2 q}^{\ell}<\mathcal{N}_{2 q}^{\ell}$. In addition, we propose an upper bound $\mathcal{N}_{2 q, \max }^{\ell}$ of $\mathcal{N}_{2 q}^{\ell}$, for all couples $(q, l)$ and all values of $N$. For $q \geq 2$, the $2 q$-th order signal subspace is spanned by the column vectors of $C_{2 q, \boldsymbol{x}}^{\ell}$. As all statistical matrices, this matrix has some redundant rows and columns due to the invariance of cumulants $C_{n_{1}, \ldots, n_{q}, x}^{n_{q+1}, \ldots, n_{2 q}}$ with respect to some permutations of index $n_{1}, \ldots, n_{2 q}$. Consequently the rank of $C_{2 q, \boldsymbol{x}}^{\ell}$ is bounded by:

$$
\mathcal{N}_{2 q, \max }^{\ell}=\frac{(N+\ell-1) !}{\ell !(N-1) !} \frac{(N+q-\ell-1) !}{(q-\ell) !(N-1) !}
$$

Regarding the previous results for $1 \leq q \leq 4$ [24], this bound can be reached for arrays with space, angular and polarization diversities. It is never reached for homogenous arrays such as arrays with space diversity only.

\section{IMPLEMENTATION AND COMPUTATIONAL COMPLEXITY}

\section{A. Recursive building of the $\mathrm{HO}$ deflation projector}

We propose in this subsection a Recursive Computational Procedure (RCP) inspired by Sorenson's work [28] in order to reduce the computational cost of $\boldsymbol{P}_{2 q}^{\ell}\left(\Theta^{p}\right)$ for every $q \geq 2$. Let $\Pi(\boldsymbol{A})$ be the rank- $p$ matrix defined by $\boldsymbol{\Pi}(\boldsymbol{A})=\boldsymbol{A}\left(\boldsymbol{A}^{\mathrm{H}} \boldsymbol{A}\right)^{-1} \boldsymbol{A}^{\mathrm{H}}$ where $\boldsymbol{A}$ is a full column rank$p$ matrix. If all sources are mutually independent, say $G=P$, then the deflation projector (10) can be built as:

$$
\left\{\begin{array}{l}
\boldsymbol{P}_{2 q}^{\ell}\left(\Theta^{0}\right)=\mathbf{I}_{N^{q}} \\
\boldsymbol{P}_{2 q}^{\ell}\left(\Theta^{p}\right)=\boldsymbol{P}_{2 q}^{\ell}\left(\Theta^{p-1}\right)-\boldsymbol{\Pi}\left(\boldsymbol{P}_{2 q}^{\ell}\left(\Theta^{p-1}\right) \boldsymbol{a}_{2 q}^{\ell}\left(\boldsymbol{\theta}_{p}\right)\right)
\end{array}\right.
$$

If all sources are mutually correlated, say $G=1$, then the deflation projector (10) can be built as:

$$
\left\{\begin{array}{l}
\boldsymbol{P}_{2}^{1}\left(\Theta^{0}\right)=\mathbf{I}_{N} \\
\boldsymbol{P}_{2}^{1}\left(\Theta^{p}\right)=\boldsymbol{P}_{2}^{1}\left(\Theta^{p-1}\right)-\boldsymbol{\Pi}\left(\boldsymbol{P}_{2}^{1}\left(\Theta^{p-1}\right) \boldsymbol{a}\left(\boldsymbol{\theta}_{p}\right)\right) \\
\boldsymbol{P}_{2 q}^{\ell}\left(\Theta^{p}\right)=\boldsymbol{P}_{2}^{1}\left(\Theta^{p}\right)^{\otimes \ell} \otimes \boldsymbol{P}_{2}^{1}\left(\Theta^{p}\right)^{* \otimes(q-\ell)}
\end{array}\right.
$$

If some sources are correlated, say $1<G<P$, then the deflation projector can be built for $1 \leq p \leq P$ as following

$$
\left\{\begin{array}{l}
\boldsymbol{P}_{2 q}^{\ell}\left(\Theta^{0}\right)=\mathbf{I}_{N^{q}} \\
\boldsymbol{P}_{2 q}^{\ell}\left(\Theta^{p}\right)=\boldsymbol{P}_{2 q}^{\ell}\left(\Theta^{p-1}\right)-\boldsymbol{\Pi}\left(\boldsymbol{P}_{2 q}^{\ell}\left(\Theta^{p-1}\right) \boldsymbol{B}_{2 q}^{\ell}\left(\Theta^{p}\right)\right)
\end{array}\right.
$$

Note that the use of the recursive projector given by (18), (19) and (20) avoids some matrix inversion and reduce therefore the computational complexity. Some simulations about this complexity are given in section V-D.

\section{B. Implementation of the methods}

The different steps of the $2 q$-D-MUSIC $(q \geq 1)$ and the $2 q$-RAP-MUSIC $(q \geq 2)$ algorithms are summarized below, when a $K$-length observation of the random vector process $\{\boldsymbol{x}(t)\}$ is available. We assume that the dimension $r_{2 q}^{\ell}$ of the initial HO signal subspace is known. The number of sources and the correlations between the sources are not known, i.e. $P, G$ and $P_{g}$ are unknown.

0 Initialization

0.1 $p=0, \widehat{\Theta}^{0}=\varnothing$ and $\boldsymbol{P}_{2 q}^{\ell}(\varnothing)=\mathbf{I}_{N^{q}}$

0.2 Estimate the $2 q$-th order statistics of the data from the realization of $\{\boldsymbol{x}(t)\}$ and compute an estimate, $\widehat{\boldsymbol{C}}_{2 q, \boldsymbol{x}}^{\ell}$, of matrix $\boldsymbol{C}_{2 q, \boldsymbol{x}}^{\ell}$

$\mathbf{0 . 3}{ }^{\mathbf{R A P}}$ Build $\widehat{\boldsymbol{E}}_{2 q, \boldsymbol{s}}^{\ell}$ by EVD of $\widehat{\boldsymbol{C}}_{2 q, \boldsymbol{x}}^{\ell}$

1 Sequential procedure to estimate the DOA $\boldsymbol{\theta}_{p}$

- if $r_{2 q}^{\ell}>0$ then go to $\mathbf{1 . 1}$ else stop the procedure

1.1 $p=p+1$

1.2 ${ }^{\mathrm{D}} \quad \widehat{\boldsymbol{C}}_{2 q, \boldsymbol{x}}^{\ell, p-1}=\boldsymbol{P}_{2 q}^{\ell}\left(\widehat{\Theta}^{p-1}\right) \widehat{\boldsymbol{C}}_{2 q, \boldsymbol{x}}^{\ell} \boldsymbol{P}_{2 q}^{\ell}\left(\widehat{\Theta}^{p-1}\right)$

1.3 $\mathbf{D}^{\mathbf{D}}$ Build a basis of the HO noise subspace $\widehat{\boldsymbol{E}}_{2 q, \boldsymbol{\nu}}^{\ell}$ from the EVD of $\widehat{\boldsymbol{C}}_{2 q, \boldsymbol{x}}^{\ell, p-1}$

1.4 $4^{\mathrm{D}}$ Build $\Upsilon\left(\boldsymbol{P}_{2 q}^{\ell}\left(\widehat{\Theta}^{p-1}\right) \boldsymbol{a}_{2 q}^{\ell}(\boldsymbol{\theta}), \widehat{\boldsymbol{E}}_{2 q, \boldsymbol{\nu}}^{\ell}\right)$

1.4 $^{\text {RAP }}$ Build $\Upsilon\left(\boldsymbol{P}_{2 q}^{\ell}\left(\widehat{\Theta}^{p-1}\right) \boldsymbol{a}_{2 q}^{\ell}(\boldsymbol{\theta}), \boldsymbol{P}_{2 q}^{\ell}\left(\widehat{\Theta}^{p-1}\right) \widehat{\boldsymbol{E}}_{2 q, \boldsymbol{s}}^{\ell}\right)$

1.5 Find the global minimizer ${ }^{\mathrm{D}}$ or maximizer ${ }^{\mathrm{RAP}} \varphi$ of the metric and its correponding minimum ${ }^{\mathrm{D}}$ or maximum ${ }^{\mathrm{RAP}} \rho$ 
- if $\left(\rho<\lambda_{1}\right)^{\mathrm{D}}$ or $\left(\rho>\lambda_{1}\right)^{\mathrm{RAP}}$ then go to $\mathbf{1 . 6}$ else stop the procedure

$$
\begin{array}{ll}
\text { 1.6 } & \hat{\boldsymbol{\theta}}_{p}=\varphi \text { and } \widehat{\Theta}^{p}=\widehat{\Theta}^{p-1} \cup\left\{\hat{\boldsymbol{\theta}}_{p}\right\} \\
\mathbf{1 . 7} & \boldsymbol{P}_{2 q}^{\ell}\left(\widehat{\Theta}^{p}\right)=\boldsymbol{\Pi}\left(\boldsymbol{P}_{2 q}^{\ell}\left(\widehat{\Theta}^{p-1}\right) \boldsymbol{a}_{2 q}^{\ell}\left(\hat{\boldsymbol{\theta}}_{p}\right)\right) \\
\mathbf{1 . 8} & r_{2 q}^{\ell}=r_{2 q}^{\ell}-1
\end{array}
$$

2 Sequential procedure to identify the sources correlated with the sources of DOA $\hat{\boldsymbol{\theta}}_{p}$ and then building the optimal deflation projector

- if $r_{2 q}^{\ell}>0$ then go to $\mathbf{2 . 1}$ else stop the procedure

2.1 $^{\mathbf{D}} \widehat{\boldsymbol{C}}_{2 q, \boldsymbol{x}}^{\ell, p}=\boldsymbol{P}_{2 q}^{\ell}\left(\widehat{\Theta}^{p}\right) \widehat{\boldsymbol{C}}_{2 q, \boldsymbol{x}}^{\ell} \boldsymbol{P}_{2 q}^{\ell}\left(\widehat{\Theta}^{p}\right)$

2.2 $\mathbf{2}^{\mathbf{D}}$ Build $\widehat{\boldsymbol{E}}_{2 q, \boldsymbol{\nu}}^{\ell}$ by EVD of $\widehat{\boldsymbol{C}}_{2 q, \boldsymbol{x}}^{\ell, p}$

2.3 ${ }^{\mathrm{D}}$ Build the generalized $2 q$-th order noise metric $\Upsilon\left(\boldsymbol{P}_{2 q}^{\ell}\left(\widehat{\Theta}^{p}\right) \tilde{\boldsymbol{a}}_{2 q}^{\ell}\left(\hat{\boldsymbol{\theta}}_{p}, \ldots, \hat{\boldsymbol{\theta}}_{p}, \boldsymbol{\theta}\right), \widehat{\boldsymbol{E}}_{2 q, \boldsymbol{\nu}}^{\ell}\right)$ for all $\boldsymbol{\theta}$

2.3 Build the generalized $2 q$-th order signal metric $\Upsilon\left(\boldsymbol{P}_{2 q}^{\ell}\left(\widehat{\Theta}^{p}\right) \tilde{\boldsymbol{a}}_{2 q}^{\ell}\left(\hat{\boldsymbol{\theta}}_{p}, \ldots, \hat{\boldsymbol{\theta}}_{p}, \boldsymbol{\theta}\right), \boldsymbol{P}_{2 q}^{\ell}\left(\widehat{\Theta}^{p} \widehat{\boldsymbol{E}}_{2 q, \boldsymbol{s}}^{\ell}\right)\right)$ for all $\boldsymbol{\theta}$

2.4 Find the global minimizer ${ }^{\mathrm{D}}$ or maximizer ${ }^{\mathrm{RAP}}$, denoted by $\varphi$, of the metric and its corresponding minimum ${ }^{\mathrm{D}}$ or maximum ${ }^{\mathrm{RAP}} \rho$

- if $\left(\rho<\lambda_{2}\right)^{\mathrm{D}}$ or $\left(\rho>\lambda_{2}\right)^{\mathrm{RAP}}$ then go to 2.5 else go to 1

2.5 Build the $M$ corresponding generalized steering vectors and order them in $D$

2.6 $\quad \boldsymbol{P}_{2 q}^{\ell}\left(\widehat{\Theta}^{p}\right)=\boldsymbol{\Pi}\left(\boldsymbol{P}_{2 q}^{\ell}\left(\widehat{\Theta}^{p}\right), \boldsymbol{D}\right)$

$2.7 \quad r_{2 q}^{\ell}=r_{2 q}^{\ell}-M$

\section{Numerical complexity}

Numerical complexity is defined here as the number of floating point operations required to execute an algorithm (flops). A flop corresponds to a multiplication followed by an addition. But, in practice, only the number of multiplications is considered since, most of the time, there are about as many (and slightly more) multiplications as additions. The numerical complexity of the proposed methods (in the case of indpendent sources) and the existing algorithm $2 q$-MUSIC are given in table II. For a given statistical order $2 q$ all algorithms have similar complexity, say $\mathcal{O}\left[N^{3 q}\right]$. Computer results will be given in V-D.

\section{COMPUTER Simulations}

The performance of the $2 q$-MUSIC (based on the noise metric), $2 q$-D-MUSIC and $2 q$-RAP-MUSIC algorithms are compared in this section for both overand underdetermined mixtures of possibly correlated sources, and in terms of numerical complexity. Two criteria [14] are computed from 500 realizations: the Probability of Non-Aberrant Results (PNAR) and the Root Mean Square Error (RMSE) between each source DOA and its estimate. A non-aberrant result is defined as a DOA corresponding to a minimum of the $2 q$-MUSIClike noise metric less than a threshold fixed at $\lambda_{1}=0.1$,

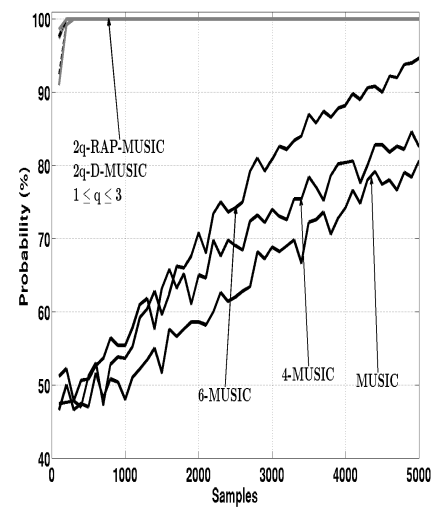

(a) minimum PNAR

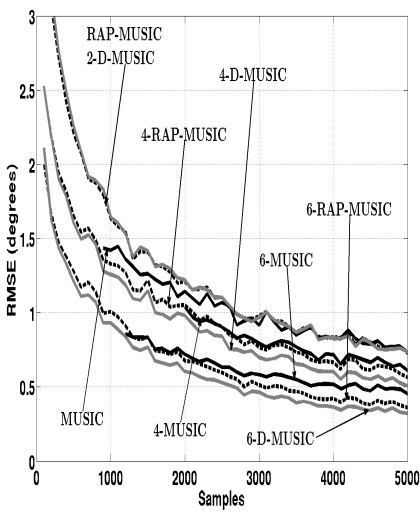

(b) maximum RMSE
Fig. 3. DOA estimation of two poorly angularly separated sources from a UCA of four sensors as a function of the number of samples without modeling error. $\Theta=\{100,105\}, \mathrm{SNR}=15 \mathrm{~dB}$.

and a maximum of the $2 q$-MUSIC-like signal metric greater than $1-\lambda_{1}=0.9$. The RMSE of the $p$-th source is computed from the non-aberrant results and defined by $\mathrm{RMSE}_{p}=\min _{p^{\prime}}\left\{\left\|\boldsymbol{\theta}_{p}-\hat{\boldsymbol{\theta}}_{p^{\prime}}\right\|\right\}$ where $\hat{\boldsymbol{\theta}}_{p^{\prime}}$ is the $p^{\prime}$ th estimated DOA. In fact, only the minimum PNAR $\left(\min _{p} \mathrm{PNAR}_{p}\right)$ and the maximum RMSE $\left(\max _{p} \mathrm{RMSE}_{p}\right)$ with respect to all sources are displayed in figures 36 , i.e only the worst estimated DOA is given. For each computer simulation, QPSK sources sampled at the symbol rate and filtered by a raised cosine of roll-off 0.3 are used [29]. All sources have a zero elevation angle and impinge on a UCA of $N$ sensors with a radius $r=0.3 \lambda$ where $\lambda$ is the wavelength of the signal.

\section{A. Overdetermined mixture of independent sources}

In this subsection, we consider $P=2$ independent sources, received by $N=4$ sensors with azimuth angles equal to $\theta_{1}=100^{\circ}$ and $\theta_{2}=105^{\circ}$, respectively. The maximum RMSE is not displayed if the corresponding minimum PNAR is around 50\%. Indeed, in such a case, at most one source is seen by the considered method, which is not satisfactory in a practical context.

Number of samples: The performance of $2 q$-RAPMUSIC, $2 q$-D-MUSIC and $2 q$-MUSIC $(1 \leq q \leq 3)$ are displayed in figure 3 as a function of the number of samples. The deflation methods are able to perfectly detect the two poorly angularly separated sources even for a small number of samples while the PNAR of $2 q$-MUSIC $(1 \leq q \leq 3)$ increases slowly to $100 \%$ (figure 3(a)). Moreover for a given value of $q \geq 2$, the deflation methods estimate the DOA a bit more precisely than the non-deflation ones (figure 3(b)). Eventually, the resolution of all the methods increases with $q$.

SNR: In figure 4, the performance of the methods is evaluated as a function of the SNR for $K=10000$ 


\begin{tabular}{||c||c|c||}
\hline \multicolumn{1}{||c||}{} & $\begin{array}{c}\text { Computational complexity } \\
N \text { : number of sensors, } P \text { : number of sources, } 2 q \text { : order of the statistical matrix with } q \geq 1, K \text { : number of data samples, } I \text { and } J: \\
\text { numbers of azimuth and elevation angles in the calibration table, respectively. }\end{array}$ \\
\hline $2 q$-MUSIC & $K(2 q-1) f_{2 q}(N)+11 N^{3 q} / 6-N^{2 q} P / 2+\left(2 N^{2 q}+N^{q}\right) I J+\mathcal{N}_{2 q}^{\ell}(q-1) I J+22(I+J)+24 I J N$ \\
\hline $2 q$-D-MUSIC & $K(2 q-1) f_{2 q}(N)+N^{3 q}(7 P / 3-11 / 6)-5 N^{2 q}(P / 2-1) / 2+\mathcal{N}_{2 q}^{\ell}(q-1) I J+22(I+J)+24 I J N$ \\
\hline $2 q$-RAP-MUSIC & $\begin{array}{l}K(2 q-1) f_{2 q}(N)+\min \left(4 N^{3 q} / 3, \frac{2 N^{3 q}+P^{2}\left(3 N^{q}-P\right)}{3}\right)+N^{2 q}\left(P^{2}+5 P-7\right) / 2+3 N^{q} P^{2}(P-1) / 2+\mathcal{N}_{2 q}^{\ell}(q-1) I J+22(I+ \\
J)+24 I J N\end{array}$ \\
\hline
\end{tabular}

TABLE II

COMPUTATIONAL COMPLEXITY OF SEVERAL CLASSICAL AND SEQUENTIAL MUSIC-LIKE METHODS FOR INDPENDENT SOURCES

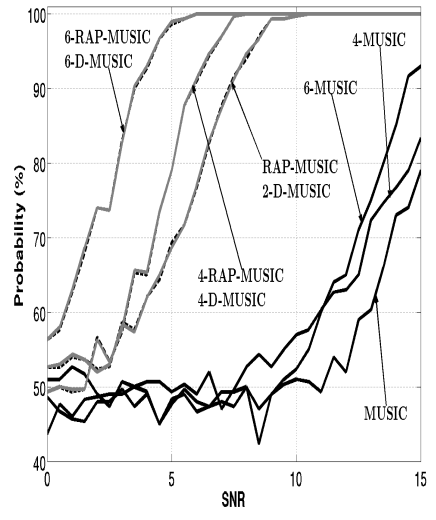

(a) minimum PNAR

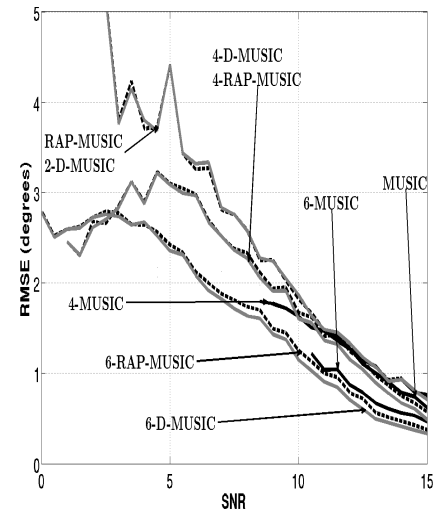

(b) maximum RMSE

Fig. 4. DOA estimation of two poorly angularly separated sources from a UCA of four sensors without modeling error as a function of the SNR. $\Theta=\{100,105\}, K=10000$ samples.

samples. For all $q$, the PNAR of the deflation methods reaches $100 \%$ faster than the PNAR of the $2 q$-MUSIC algorithm. Regarding the RMSE, the superiority of both SixO sequential approaches over the three SO-MUSIClike techniques clearly appears in figure 4(b) for SNR values from 0 to $12.5 \mathrm{~dB}$.

Modeling errors: In operational contexts, for given choices of array of sensors and algorithm, the performance of the latter is mainly controlled by modeling errors such as array calibration errors or phase and amplitude residual mismatches between reception chains. For this reason, it is important to compute the performance of the $2 q$-MUSIC, $2 q$-D-MUSIC and $2 q$-RAP-MUSIC in the presence of modeling errors, showing their behavior in such contexts. First, the scenario of figure 3 with modeling errors of variance $10^{-4}[14]$ is considered and computer results are presented in figure 5. The PNAR of the $2 q$-MUSIC methods does not converge at all and fluctuates around $50 \%$. On the contrary, the deflation approaches show a PNAR of $100 \%$ from a low number of samples (less than 500). Regarding the RMSE, the gap of performance between the $\mathrm{SO}, \mathrm{FO}$ and $\mathrm{SixO}$ sequential approaches is increased in comparison with

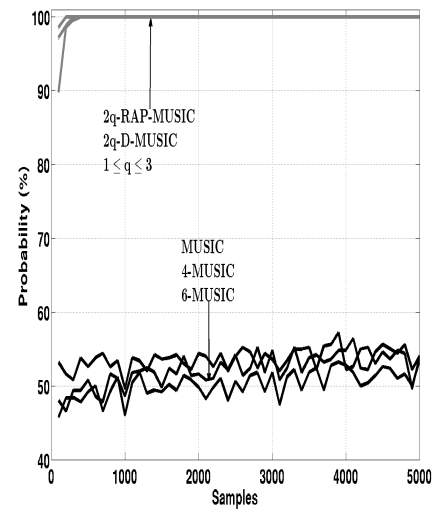

(a) minimum PNAR

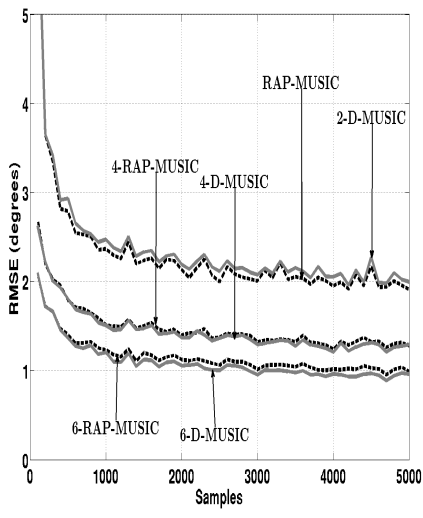

(b) maximum RMSE
Fig. 5. DOA estimation of two poorly angularly separated sources from a UCA of four sensors as a function of the number of samples with modeling errors of variance $10^{-4} . \Theta=\{100,105\}$, SNR $=15$ dB.

figure 3. Second, the performance of the methods are displayed in figure 6 as a function of the variance of modeling errors for $K=10000$ samples. It appears that the $2 q$-D-MUSIC and $2 q$-RAP-MUSIC methods are the most robust with respect to an increasing variance of modeling errors. Among the latter, the HO methods have a quasi-perfect PNAR, while the PNAR of SO techniques decreases from a variance of $2.10^{-4}$ (figure 6(a)). The $2 q$-MUSIC approaches are inefficient as soon as the variance of modeling errors is greater than $10^{-4}$. For this reason, their RMSE are not displayed. The RMSE of the sequential algorithms shows a performance that increases with $q$ (figure 6(b)).

\section{B. Underdetermined mixture of independent sources}

We consider $P=5$ independent sources, received by $N=4$ sensors, with azimuth angles equal to $\theta_{1}=20^{\circ}$, $\theta_{2}=47.5^{\circ}, \theta_{3}=100^{\circ}, \theta_{4}=200^{\circ}$ and $\theta_{5}=227.5^{\circ}$, respectively. The performance of $2 q$-RAP-MUSIC, $2 q$ D-MUSIC and $2 q$-MUSIC $(2 \leq q \leq 3)$ are displayed in figure 7 as a function of the number of samples without modeling errors. It appears that the PNAR of 


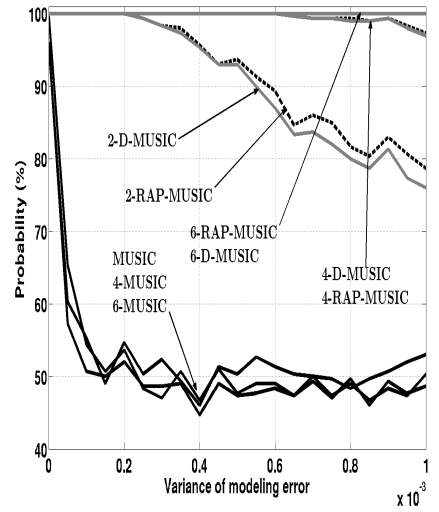

(a) minimum PNAR

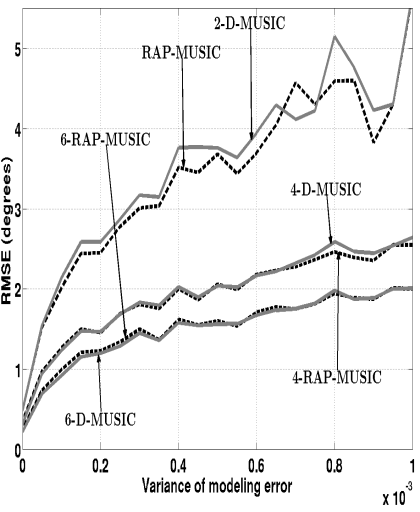

(b) maximum RMSE

Fig. 6. DOA estimation of two poorly angularly separated sources from a UCA of four sensors as a function of the variance of modeling errors. $\Theta=\{100,105\}, K=10000, \mathrm{SNR}=15 \mathrm{~dB}$.

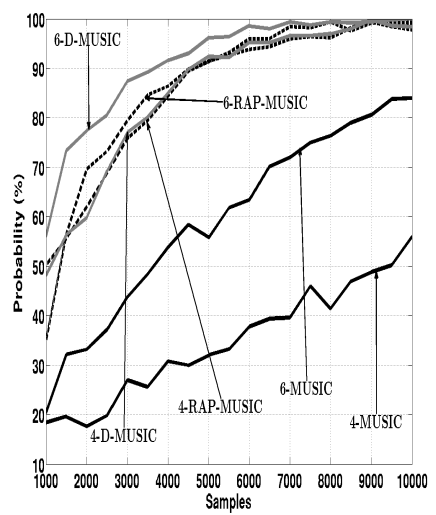

(a) minimum PNAR

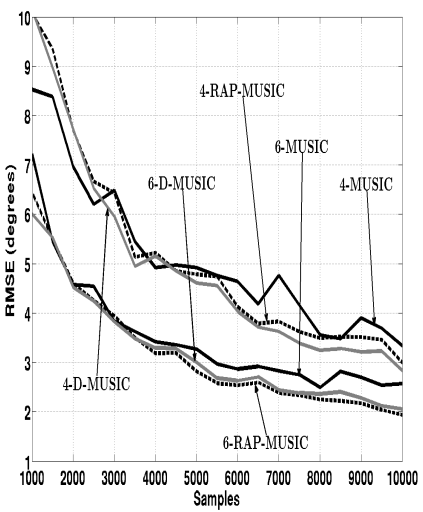

(b) maximum RMSE

Fig. 7. DOA estimation of five sources from a UCA of four sensors as a function of the number of samples without modeling error. $\Theta=$ $\{20,47.5,100,200,227.5\}, \mathrm{SNR}=15 \mathrm{~dB}$.

the deflation methods increases faster than the PNAR of the other algorithms (figure 7(a)). As shown as in the overdetermined case the higher the statistical order $2 q$, the lower the RMSE (figure 7(b)).

\section{Underdetermined mixture of correlated sources}

The performance of the 4-RAP-MUSIC-1 and 4-RAPMUSIC-2 methods are compared to the performance of the existing algorithm 4-MUSIC in the presence of an underdetermined mixture of correlated sources. 4-RAP-MUSIC-1 uses the optimal deflation projector based on the optimzation of the generalized $2 q$-MUSIC metric while 4-RAP-MUSIC-2 uses the deflation projector (9) optimal only for independent sources, i.e. for $\boldsymbol{B}_{2 q}^{\ell}\left(\Theta^{p}\right)=\left[\boldsymbol{a}_{2 q}^{\ell}\left(\boldsymbol{\theta}_{1}\right), \ldots, \boldsymbol{a}_{2 q}^{\ell}\left(\boldsymbol{\theta}_{p}\right)\right]$. As both proposed sequential algorithms $2 q$-D-MUSIC and $2 q$-RAP-MUSIC have a close performance, we only show the results of 4RAP-MUSIC-1 and 4-RAP-MUSIC-2. A UCA of $N=3$ sensors is used in order to estimate $P=3$ source DOA's

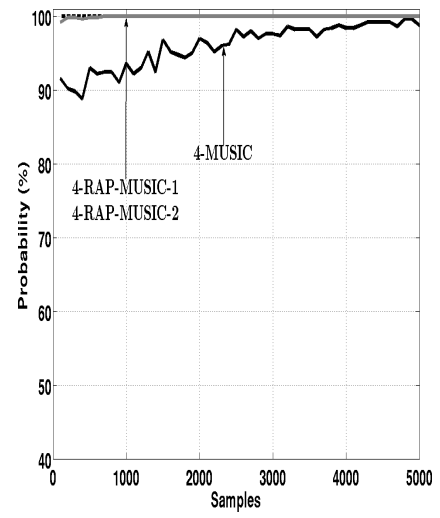

(a) minimum PNAR

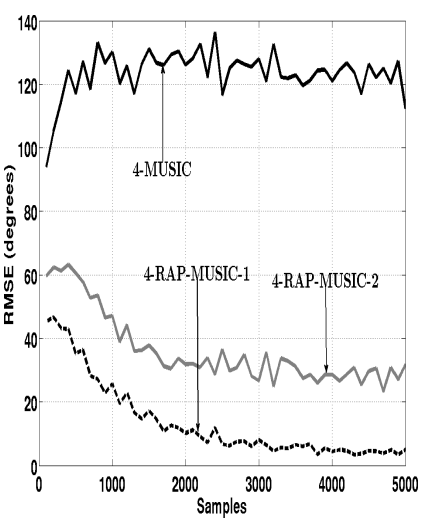

(b) maximum RMSE
Fig. 8. DOA estimation of three sources from a UCA of three sensors as a function of the number of samples without modeling error. $\Theta=\{50,100,105\}$, the correlation between sources 1 and 2 is set to 0.7 , the third source is independent, $\mathrm{SNR}=15 \mathrm{~dB}$.

$\Theta_{1}=\{50,100\}$ and $\Theta_{2}=\{105\}$, say sources 1 and 2 are correlated, source 3 is independent. In order to set the correlation between the sources, we build the $P$ processes $\left\{s_{p}(t)\right\}$ as:

$$
s_{p}(t)=\sum_{k \in \mathbb{Z}}\left(\alpha_{k}^{(p)} a_{k}+\left(1-\alpha_{k}^{(p)}\right) b_{k}^{(p)}\right) h(t-k T)
$$

where $T$ is the symbol rate, $a_{k}$ and the $P$ variables $b_{k}^{(p)}$ are independent with equiprobable values in $\{-\mathrm{i}, \mathrm{i},-1,1\}, h$ is the pattern of the modulation defined as a Nyquist filter and the $P$ independent random variables $\alpha_{k}^{(p)}$ have the density $P\left(\alpha_{k}^{(p)}=1\right)=1-$ $P\left(\alpha_{k}^{(p)}=0\right)=\beta_{p}$. The values of the $P$ parameters $\beta_{p}$ set the intercorrelation between the $P$ sources. Indeed, for the processes $\left\{s_{i}(t)\right\}$ and $\left\{s_{j}(t)\right\}, i \neq j$, we have $\operatorname{Cov}\left(s_{i}(t), s_{j}(t)^{*}\right)=\beta_{i} \beta_{j} \operatorname{Var}\left(a_{k}\right)$. For the source configuration described at the beginning of this subsection, the three parameters $\beta_{p}$ are set to $\beta_{1}=1, \beta_{2}=\beta$ and $\beta_{3}=0$. Thus, the correlation between sources 1 and 2 is equal to $\beta$ (since $\operatorname{Var}\left(a_{k}\right)=1$ ), while the source 3 is independent of both sources 1 and 2 .

We give in figure 8 the PNAR and RMSE of 4-RAPMUSIC-1, 4-RAP-MUSIC-2 and 4-MUSIC as a function of the number of samples for $\beta=0.7$. All methods have a good enough PNAR. The RMSE of the proposed 4RAP-MUSIC-1 algorithm seems to converge to 0 while the RMSE of 4-RAP-MUSIC-2 and 4-MUSIC remain over 20 and 100 degrees, respectively.

The PNAR and RMSE of 4-RAP-MUSIC-1, 4-RAPMUSIC-2 and 4-MUSIC as a function of the correlation coefficient $\beta$ are displayed in figure 9. The PNAR of 4MUSIC is ranged from 95 to $100 \%$ while the PNAR of both sequential methods 4-RAP-MUSIC-1 and 4-RAPMUSIC-2 is equal to $100 \%$. The RMSE of the 4-MUSIC 


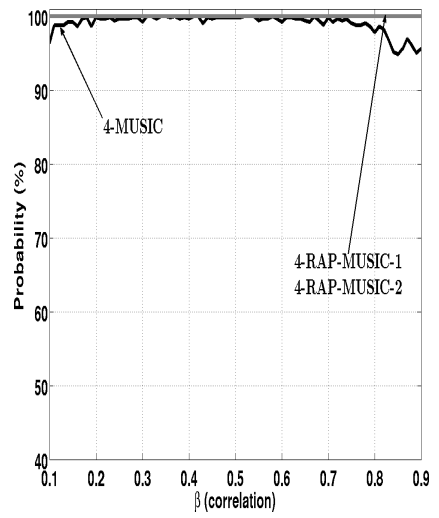

(a) minimum PNAR

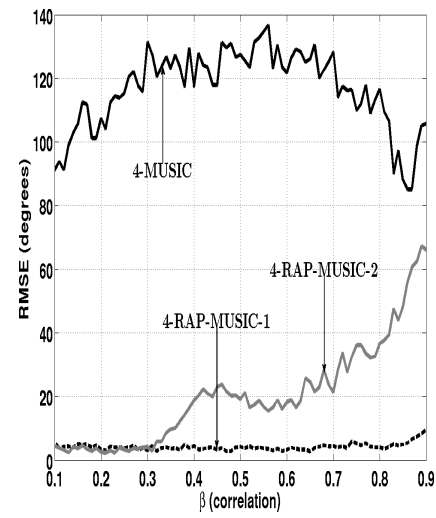

(b) maximum RMSE
Fig. 9. DOA estimation of three sources from a UCA of three sensors as a function of the correlation between sources 1 and 2 . $\Theta=\{50,100,105\}$, the third source is independent, $\mathrm{SNR}=15 \mathrm{~dB}$ and $K=5000$ samples.

remains over 80 degrees, showing that this method is not efficient in such a situation. The 4-RAP-MUSIC-1 and 4-RAP-MUSIC-2 algorithms show similar RMSE (about 5 degrees) for $\beta$ varying from 0.1 to 0.3 . For $\beta$ beyond 0.3 , the performance of 4-RAP-MUSIC- 2 degraduates as $\beta$ increases, while the RMSE of 4-RAP-MUSIC- 1 is very stable.

\section{Study of numerical complexity}

The performance of $2 q$-D-MUSIC, $2 q$-RAP-MUSIC and $2 q$-MUSIC are studied as a function of the complexity in the case of independent sources. Figure 10 shows the minimum numerical complexity of the deflation projector built recursively $\left(\boldsymbol{P}_{2}\right)$ and not recursively $\left(\boldsymbol{P}_{1}\right)$, and the minimum computational complexity of the classical and sequential MUSIC-like methods as a function of the number $P$ of sources. For each value of $P$ the minimum number $N_{\min }$ of sensors of a UCA is used such that the identifiability condition of each method is still valid, hence the term "minimum complexity". Thus, we have $N_{\min }=P+1$ for MUSIC, 2-D-MUSIC, RAP-MUSIC and $N_{\text {min }} \leq P+1 \leq \mathcal{N}_{2 q}^{\ell}$ for the other algorithms allowing for an identification of underdetermined mixtures. The number of points, $I$ and $J$, of the grid $\{\boldsymbol{\theta}\}$ is equal to 360 and 1 , respectively. Figure 10(a) shows that the recursive procedure proposed in section IV-A should be preferred in order to reduce the numerical complexity of $\boldsymbol{P}_{2 q}^{\ell}\left(\Theta^{p}\right)$. Moreover, it is shown in figure 10(b) that the non-sequential MUSIClike methods are less expensive than the deflation ones, and that the computational complexity increases with the statistical order $2 q(q \geq 1)$, as expected. The figure 11 shows the performance of deflation and non-sequential MUSIC-like methods as a function of the numerical
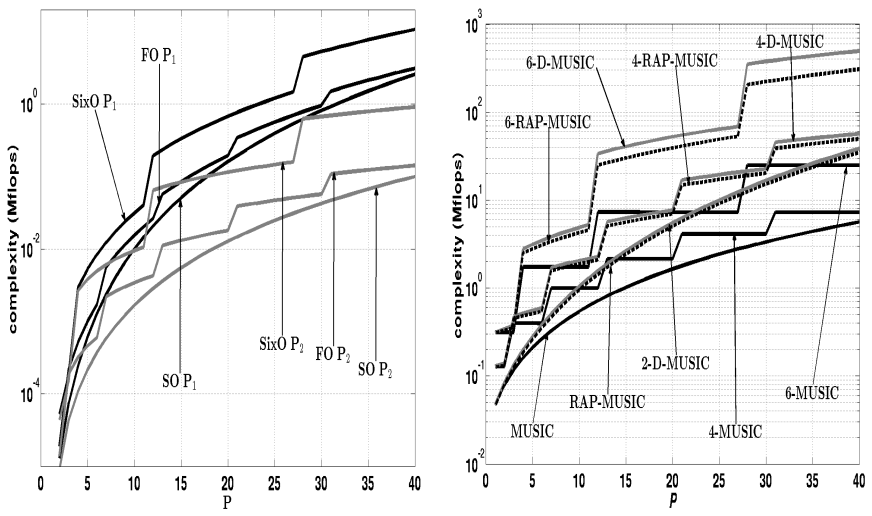

(a) Recursive $\left(P_{2}\right)$ versus nonrecursive $\left(P_{1}\right)$ procedures to compute projectors

(b) Comparison between sequential/non-sequential $\mathrm{SO} / \mathrm{HO}$ MUSIC-like methods

Fig. 10. Minimum numerical complexity as a function of $P$

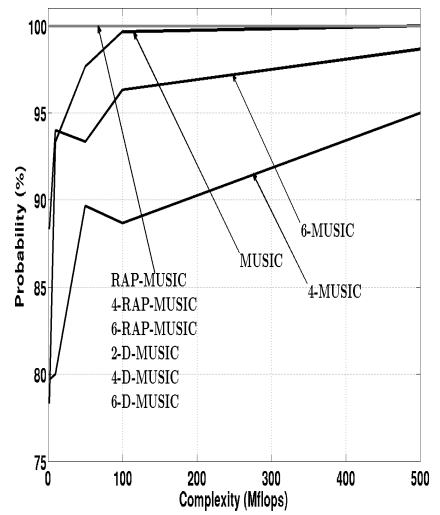

(a) mean of PNAR

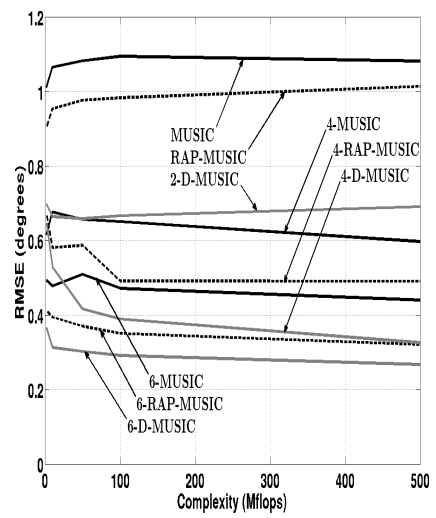

(b) mean of $R M S E_{p}$
Fig. 11. Performance of methods as a function of the complexity

complexity for a finite number of samples $(K=5000)$, a finite SNR $(15 \mathrm{~dB})$ and two angularly close $\left(100^{\circ}\right.$ and $105^{\circ}$ ) sources impinging on a UCA. Note that the wanted computational complexity is obtained by varying the number of sensors of each method as specified in table III. When a method must be chosen for a

\begin{tabular}{|l|c|c|c|c|c|c|}
\hline Complexity & 2.0 & 8.7 & 29.8 & 87.5 & 228.9 & 551.6 \\
\hline MUSIC & 23 & 51 & 96 & 164 & 259 & 390 \\
4-MUSIC & 3 & 5 & 7 & 9 & 11 & 14 \\
6-MUSIC & 3 & 4 & 5 & 6 & 7 & 8 \\
\hline \hline 2-D-MUSIC & 22 & 47 & 86 & 143 & 219 & 320 \\
4-D-MUSIC & 3 & 5 & 6 & 9 & 11 & 13 \\
6-D-MUSIC & 3 & 4 & 5 & 5 & 6 & 7 \\
\hline \hline RAP-MUSIC & 8 & 22 & 49 & 92 & 157 & 251 \\
4-RAP-MUSIC & 3 & 5 & 7 & 9 & 11 & 14 \\
6-RAP-MUSIC & 3 & 4 & 5 & 6 & 7 & 8 \\
\hline
\end{tabular}

TABLE III

NUMBER OF SENSORS CORRESPONDING TO A GIVEN COMPLEXITY (IN MFLOPS) FOR SEVERAL METHODS 
given problem, a compromise between complexity and performance has to be made. The low cost MUSIC method has a poor resolution and is then not able to differentiate angularly close sources. Consequently if a high resolution is needed by the user, a $\mathrm{HO}$ deflation method is required in order to solve the problem with a high probability.

\section{CONCLUSION}

We proposed some extensions of the sequential MUSIC-like algorithms to $\mathrm{HO}$ statistics called $2 q$-DMUSIC and $2 q$-RAP-MUSIC $(q \geq 2)$. We introduced an optimal HO deflation orthogonal projector able to deal with both independent and correlated sources. This projector is built thanks to a generalized $2 q$-MUSIC metric capable of identifying the actual correlated sources. We reduced the computational complexity by building recursively the projector at each step of the algorithms. We also give a generalized upper bound of the rank of statistical matrices. As shown by computer results, $2 q$-D-MUSIC and $2 q$-RAP-MUSIC $(q \geq 2)$ are i) able to process underdetermined mixtures of independent or correlated sources and ii) robust to modeling errors. Moreover, the novel methods show a higher accuracy for localizing very angularly close sources than the classical $2 q$-MUSIC methods. In addition, an analysis of the proposed methods was performed in terms of numerical complexity. In spite of the high computational loads required be the use of HO statistics, it confirms the fact that, when a high resolution is required, a $\mathrm{HO}$ deflation technique should be used in order to find source DOA's with a high probability. In addition, the current processors and RAM sizes are able to deal with it in a reasonable time. At all events, a forthcoming work will include a way of decreasing the computational cost of our methods by reducing the dimensions of the statistical matrix.

\section{APPENDIX}

A. Proof that equation (10) defines an orthogonal MPO along a subspace containing $\operatorname{Span}\left\{\boldsymbol{B}_{2 q}^{\ell}\left(\Theta^{p}\right)\right\}$

Let's recall what characteristic features distinguish an orthogonal MPO from another matrix [27, page 433]. First, matrix $\boldsymbol{P}$ will be a MPO iff $\boldsymbol{P}$ is idempotent, say iff $\boldsymbol{P P}=\boldsymbol{P}$. Next, a MPO will be orthogonal iff it is Hermitian. Eventually, given $\boldsymbol{P}$ and $\boldsymbol{A}$ an MPO and a matrix such that the product $\boldsymbol{P} \boldsymbol{A}$ exists, the latter will vanish iff $\operatorname{Span}\{\boldsymbol{A}\}$ is included in the null-space of $\boldsymbol{P}$. Then, in order to show that $\boldsymbol{P}_{2 q}^{\ell}\left(\Theta^{p}\right)$ is an orthogonal MPO whose null-space includes $\operatorname{Span}\left\{\boldsymbol{B}_{2 q}^{\ell}\left(\Theta^{p}\right)\right\}$ is a subspace of its null-space, we have to prove that it is idempotent, Hermitian and the following equality $\boldsymbol{P}_{2 q}^{\ell}\left(\Theta^{p}\right) \boldsymbol{B}_{2 q}^{\ell}\left(\Theta^{p}\right)=\mathbf{0}$.

Firstly, from equation (10), we have:

$$
\begin{aligned}
& \boldsymbol{P}_{2 q}^{\ell}\left(\Theta^{p}\right) \boldsymbol{P}_{2 q}^{\ell}\left(\Theta^{p}\right)= \\
& \left(\boldsymbol{P}_{2}^{1}\left(\Theta^{p}\right)^{\otimes(q-\ell)} \otimes \boldsymbol{P}_{2}^{1}\left(\Theta^{p}\right)^{* \otimes \ell}\right)\left(\boldsymbol{P}_{2}^{1}\left(\Theta^{p}\right)^{\otimes(q-\ell)} \otimes \boldsymbol{P}_{2}^{1}\left(\Theta^{p}\right)^{* \otimes \ell}\right)
\end{aligned}
$$

Thus, using properties of the Kronecker product [30, equation (2.5)], we get:

$$
\begin{aligned}
& \boldsymbol{P}_{2 q}^{\ell}\left(\Theta^{p}\right) \boldsymbol{P}_{2 q}^{\ell}\left(\Theta^{p}\right)= \\
& \quad\left(\boldsymbol{P}_{2}^{1}\left(\Theta^{p}\right) \boldsymbol{P}_{2}^{1}\left(\Theta^{p}\right)\right)^{\otimes(q-\ell)} \otimes\left(\boldsymbol{P}_{2}^{1}\left(\Theta^{p}\right) \boldsymbol{P}_{2}^{1}\left(\Theta^{p}\right)\right)^{* \otimes \ell}
\end{aligned}
$$

Since $\boldsymbol{P}_{2}^{1}\left(\Theta^{p}\right)$ is a MPO, it is idempotent and then we get:

$\boldsymbol{P}_{2 q}^{\ell}\left(\Theta^{p}\right) \boldsymbol{P}_{2 q}^{\ell}\left(\Theta^{p}\right)=\boldsymbol{P}_{2}^{1}\left(\Theta^{p}\right)^{\otimes(q-\ell)} \otimes \boldsymbol{P}_{2}^{1}\left(\Theta^{p}\right)^{* \otimes \ell}=\boldsymbol{P}_{2 q}^{\ell}\left(\Theta^{p}\right)$

which shows that matrix $\boldsymbol{P}_{2 q}^{\ell}\left(\Theta^{p}\right)$ is a MPO.

Second, from equation (10), we have:

$$
\boldsymbol{P}_{2 q}^{\ell}\left(\Theta^{p}\right)^{\mathrm{H}}=\left(\boldsymbol{P}_{2}^{1}\left(\Theta^{p}\right)^{\otimes(q-\ell)} \otimes \boldsymbol{P}_{2}^{1}\left(\Theta^{p}\right)^{* \otimes \ell}\right)^{\mathrm{H}}
$$

using properties of the Kronecker product [30, equation (2.6)], we get:

$$
\boldsymbol{P}_{2 q}^{\ell}\left(\Theta^{p}\right)^{\mathrm{H}}=\left(\boldsymbol{P}_{2}^{1}\left(\Theta^{p}\right)^{\mathrm{H}}\right)^{\otimes(q-\ell)} \otimes\left(\boldsymbol{P}_{2}^{1}\left(\Theta^{p}\right)^{\mathrm{H}}\right)^{* \otimes \ell}
$$

Since $\boldsymbol{P}_{2}^{1}\left(\Theta^{p}\right)$ is an orthogonal MPO, it is Hermitian and then we have:

$$
\boldsymbol{P}_{2 q}^{\ell}\left(\Theta^{p}\right)^{\mathrm{H}}=\left(\boldsymbol{P}_{2}^{1}\left(\Theta^{p}\right)\right)^{\otimes(q-\ell)} \otimes\left(\boldsymbol{P}_{2}^{1}\left(\Theta^{p}\right)\right)^{* \otimes \ell}=\boldsymbol{P}_{2 q}^{\ell}\left(\Theta^{p}\right)
$$

Consequently, the MPO $\boldsymbol{P}_{2 q}^{\ell}\left(\Theta^{p}\right)$ is Hermitian, say orthogonal.

Finally, let's prove that the null-space of $\boldsymbol{P}_{2 q}^{\ell}\left(\Theta^{p}\right)$ contains $\operatorname{Span}\left\{\boldsymbol{B}_{2 q}^{\ell}\left(\Theta^{p}\right)\right\}$. We get:

$$
\begin{aligned}
{\left[\boldsymbol{P}_{2 q}^{\ell}\left(\Theta^{p}\right) \boldsymbol{B}_{2 q}^{\ell}\left(\Theta^{p}\right)\right.} & \left., \boldsymbol{P}_{2 q}^{\ell}\left(\Theta^{p}\right) \boldsymbol{A}_{2 q}^{\ell}\left(\bar{\Theta}^{p}\right)\right] \\
& =\boldsymbol{P}_{2 q}^{\ell}\left(\Theta^{p}\right)\left[\boldsymbol{B}_{2 q}^{\ell}\left(\Theta^{p}\right), \boldsymbol{A}_{2 q}^{\ell}\left(\bar{\Theta}^{p}\right)\right] \\
& =\boldsymbol{P}_{2 q}^{\ell}\left(\Theta^{p}\right) \boldsymbol{A}_{2 q}^{\ell}(\Theta) \boldsymbol{\Sigma}_{2 q}
\end{aligned}
$$

where $\boldsymbol{\Sigma}_{2 q}$ is a particular permutation matrix, and more particularly:

$$
\begin{aligned}
& {\left[\boldsymbol{P}_{2 q}^{\ell}\left(\Theta^{p}\right) \boldsymbol{B}_{2 q}^{\ell}\left(\Theta^{p}\right), \boldsymbol{P}_{2 q}^{\ell}\left(\Theta^{p}\right) \boldsymbol{A}_{2 q}^{\ell}\left(\bar{\Theta}^{p}\right)\right]=} \\
& \left(\boldsymbol{P}_{2}^{1}\left(\Theta^{p}\right)^{\otimes(q-\ell)} \otimes \boldsymbol{P}_{2}^{1}\left(\Theta^{p}\right)^{* \otimes \ell}\right)\left(\boldsymbol{A}(\Theta)^{\otimes(q-\ell)} \otimes\left(\boldsymbol{A}(\Theta)^{* \otimes \ell}\right) \boldsymbol{\Sigma}_{2 q}\right.
\end{aligned}
$$

Using properties of the Kronecker product again [30, equation (2.5)], we get:

$$
\begin{aligned}
{\left[\boldsymbol{P}_{2 q}^{\ell}\left(\Theta^{p}\right)\right.} & \left.\boldsymbol{B}_{2 q}^{\ell}\left(\Theta^{p}\right), \boldsymbol{P}_{2 q}^{\ell}\left(\Theta^{p}\right) \boldsymbol{A}_{2 q}^{\ell}\left(\bar{\Theta}^{p}\right)\right] \\
= & \left(\left(\boldsymbol{P}_{2}^{1}\left(\Theta^{p}\right) \boldsymbol{A}(\Theta)\right)^{\otimes(q-\ell)} \otimes\left(\boldsymbol{P}_{2}^{1}\left(\Theta^{p}\right) \boldsymbol{A}(\Theta)\right)^{* \otimes \ell}\right) \boldsymbol{\Sigma}_{2 q} \\
= & \left(\left(\boldsymbol{P}_{2}^{1}\left(\Theta^{p}\right)\left[\boldsymbol{A}\left(\Theta^{p}\right), \boldsymbol{A}\left(\bar{\Theta}^{p}\right)\right] \boldsymbol{\Sigma}_{2}\right){ }^{\otimes(q-\ell)}\right. \\
& \left.\otimes\left(\boldsymbol{P}_{2}^{1}\left(\Theta^{p}\right)\left[\boldsymbol{A}\left(\Theta^{p}\right), \boldsymbol{A}\left(\bar{\Theta}^{p}\right)\right] \boldsymbol{\Sigma}_{2}\right)^{* \otimes \ell}\right) \boldsymbol{\Sigma}_{2 q}
\end{aligned}
$$


where $\boldsymbol{\Sigma}_{2}$ is a permutation matrix such that $\boldsymbol{\Sigma}_{2}^{\otimes q}=$ $\boldsymbol{\Sigma}_{2 q}$. Now $\boldsymbol{P}_{2}^{1}\left(\Theta^{p}\right)$ is the orthogonal MPO along $\operatorname{Span}\left\{\boldsymbol{A}\left(\Theta^{p}\right)\right\}$, then we obtain:

$$
\begin{aligned}
& {\left[\boldsymbol{P}_{2 q}^{\ell}\left(\Theta^{p}\right) \boldsymbol{B}_{2 q}^{\ell}\left(\Theta^{p}\right), \boldsymbol{P}_{2 q}^{\ell}\left(\Theta^{p}\right) \boldsymbol{A}_{2 q}^{\ell}\left(\bar{\Theta}^{p}\right)\right]} \\
& =\left(\left(\left[\mathbf{0}, \boldsymbol{P}_{2}^{1}\left(\Theta^{p}\right) \boldsymbol{A}\left(\bar{\Theta}^{p}\right)\right] \boldsymbol{\Sigma}_{2}\right)^{\otimes(q-\ell)}\right. \\
& \left.\otimes\left(\left[\mathbf{0}, \boldsymbol{P}_{2}^{1}\left(\Theta^{p}\right) \boldsymbol{A}\left(\bar{\Theta}^{p}\right)\right] \boldsymbol{\Sigma}_{2}\right)^{* \otimes \ell}\right) \boldsymbol{\Sigma}_{2 q}
\end{aligned}
$$

Using properties of the Kronecker product again [30, equation (2.5)], we get:

$$
\begin{aligned}
& {\left[\boldsymbol{P}_{2 q}^{\ell}\left(\Theta^{p}\right) \boldsymbol{B}_{2 q}^{\ell}\left(\Theta^{p}\right), \boldsymbol{P}_{2 q}^{\ell}\left(\Theta^{p}\right) \boldsymbol{A}_{2 q}^{\ell}\left(\bar{\Theta}^{p}\right)\right]} \\
& =\left(\left[\mathbf{0}, \boldsymbol{P}_{2}^{1}\left(\Theta^{p}\right) \boldsymbol{A}\left(\bar{\Theta}^{p}\right)\right]^{\otimes(q-\ell)} \otimes\left[\mathbf{0}, \boldsymbol{P}_{2}^{1}\left(\Theta^{p}\right) \boldsymbol{A}\left(\bar{\Theta}^{p}\right)\right]^{* \otimes \ell}\right) \boldsymbol{\Sigma}_{2}^{\otimes q} \boldsymbol{\Sigma}_{2 q} \\
& =\left[\mathbf{0},\left(\boldsymbol{P}_{2}^{1}\left(\Theta^{p}\right) \boldsymbol{A}\left(\bar{\Theta}^{p}\right)\right)^{\otimes(q-\ell)} \otimes\left(\boldsymbol{P}_{2}^{1}\left(\Theta^{p}\right) \boldsymbol{A}\left(\bar{\Theta}^{p}\right)\right)^{* \otimes \ell}\right] \boldsymbol{\Sigma}_{2}^{\otimes q} \boldsymbol{\Sigma}_{2 q} \\
& =\left[\mathbf{0}, \boldsymbol{P}_{2 q}^{\ell}\left(\Theta^{p}\right) \boldsymbol{A}_{2 q}^{\ell}\left(\bar{\Theta}^{p}\right)\right] \boldsymbol{\Sigma}_{2}^{\otimes q} \boldsymbol{\Sigma}_{2 q} \\
& =\left[\mathbf{0}, \boldsymbol{P}_{2 q}^{\ell}\left(\Theta^{p}\right) \boldsymbol{A}_{2 q}^{\ell}\left(\bar{\Theta}^{p}\right)\right]
\end{aligned}
$$

Recall that $\boldsymbol{\Sigma}_{2}^{\otimes q}=\boldsymbol{\Sigma}_{2 q}$ and the product of the permutation $\boldsymbol{\Sigma}_{2 q}$ by itself is equal to the identity matrix. As a result, equalizing both sides of the latter equation, we get $\boldsymbol{P}_{2 q}^{\ell}\left(\Theta^{p}\right) \boldsymbol{B}_{2 q}^{\ell}\left(\Theta^{p}\right)=\mathbf{0}$. Hence the result.

\section{B. Equivalence between $\mathrm{HO}$ noise and signal metrics}

According to (6), we get:

$$
\Upsilon\left(\boldsymbol{a}_{2 q}^{\ell}(\boldsymbol{\theta}), \boldsymbol{E}_{2 q, \boldsymbol{\nu}}^{\ell}\right)=\frac{\boldsymbol{a}_{2 q}^{\ell}(\boldsymbol{\theta})^{\mathrm{H}} \boldsymbol{\Pi}_{2 q, \boldsymbol{\nu}}^{\ell} \boldsymbol{a}_{2 q}^{\ell}(\boldsymbol{\theta})}{\boldsymbol{a}_{2 q}^{\ell}(\boldsymbol{\theta})^{\mathrm{H}} \boldsymbol{a}_{2 q}^{\ell}(\boldsymbol{\theta})}
$$

where $\boldsymbol{\Pi}_{2 q, \boldsymbol{\nu}}^{\ell}=\boldsymbol{E}_{2 q, \boldsymbol{\nu}}^{\ell}\left(\boldsymbol{E}_{2 q, \boldsymbol{\nu}^{H}}^{\ell} \boldsymbol{E}_{2 q, \boldsymbol{\nu}}^{\ell}\right)^{-1} \boldsymbol{E}_{2 q, \boldsymbol{\nu}^{H}}^{\ell}$ denotes the orthogonal MPO onto the $2 q$-th order noise subspace. Let $\boldsymbol{\Pi}_{2 q, s}^{\ell}=\boldsymbol{E}_{2 q, \boldsymbol{s}}^{\ell}\left(\boldsymbol{E}_{2 q, s^{\ell}}^{\ell} \boldsymbol{E}_{2 q, \boldsymbol{s}}^{\ell}\right)^{-1} \boldsymbol{E}_{2 q, s^{H}}^{\ell}$ be the orthogonal MPO onto the $2 q$-th order signal subspace. Since the $2 q$ th order noise and signal subspaces are two orthogonal complementary subspaces by construction, MPO's $\Pi_{2 q, \nu}^{\ell}$ and $\boldsymbol{\Pi}_{2 q, s}^{\ell}$ are related as following: $\boldsymbol{\Pi}_{2 q, \nu}^{\ell}=\mathbf{I}_{N^{q}}-\boldsymbol{\Pi}_{2 q, \boldsymbol{s}}^{\ell}$. Consequently, we have:

$$
\begin{aligned}
\Upsilon\left(\boldsymbol{a}_{2 q}^{\ell}(\boldsymbol{\theta}), \boldsymbol{E}_{2 q, \boldsymbol{\nu}}^{\ell}\right) & =\frac{\boldsymbol{a}_{2 q}^{\ell}(\boldsymbol{\theta})^{\mathrm{H}}\left(\mathbf{I}_{N^{q}}-\boldsymbol{\Pi}_{2 q, \boldsymbol{s}}^{\ell}\right) \boldsymbol{a}_{2 q}^{\ell}(\boldsymbol{\theta})}{\boldsymbol{a}_{2 q}^{\ell}(\boldsymbol{\theta})^{\mathrm{H}} \boldsymbol{a}_{2 q}^{\ell}(\boldsymbol{\theta})} \\
& =\frac{\boldsymbol{a}_{2 q}^{\ell}(\boldsymbol{\theta})^{\mathrm{H}} \boldsymbol{a}_{2 q}^{\ell}(\boldsymbol{\theta})}{\boldsymbol{a}_{2 q}^{\ell}(\boldsymbol{\theta})^{\mathrm{H}} \boldsymbol{a}_{2 q}^{\ell}(\boldsymbol{\theta})}-\frac{\boldsymbol{a}_{2 q}^{\ell}(\boldsymbol{\theta})^{\mathrm{H}} \boldsymbol{\Pi}_{2 q, s}^{\ell} \boldsymbol{a}_{2 q}^{\ell}(\boldsymbol{\theta})}{\boldsymbol{a}_{2 q}^{\ell}(\boldsymbol{\theta})^{\mathrm{H}} \boldsymbol{a}_{2 q}^{\ell}(\boldsymbol{\theta})} \\
& =1-\Upsilon\left(\boldsymbol{a}_{2 q}^{\ell}(\boldsymbol{\theta}), \boldsymbol{E}_{2 q, \boldsymbol{s}}^{\ell}\right)
\end{aligned}
$$

\section{REFERENCES}

[1] I. MERLET, "Dipole modeling of interictal and ictal EEG and MEG paroxysms," Epileptic Disord, no. Special Issue, pp. 1136, October 2001.

[2] C. PLUMMER, A. S. HARVEY, and M. COOK, "EEG source localization in focal epilepsy: Where are we now?" Epilepsia, October 2007.

[3] S. MIRON, N. LE BIHAN, and J. I. MARS, "Vector-sensor MUSIC for polarized seismic sources localization," EURASIP Journal on Applied Signal Processing, vol. 10, pp. 74-84, October 2005.

[4] H. KRIM and M. VIBERG, "Two decades of signal processing: The parametric approach," IEEE Signal Processing Magazine, vol. 13, pp. 67-94, July 1996.

[5] G. BIENVENU and L. KOPP, "Optimality of high resolution array processing using the eigensystem approach," IEEE Trans. Acou. Speech and Sign. Proc., vol. 31, no. 5, pp. 1235-1247, October 1983.

[6] R. O. SCHMIDT, "Multiple emitter location and signal parameter estimation," IEEE Transactions On Antennas Propagation, vol. 34, no. 3, pp. 276-280, March 1986, reprint of the original 1979 paper from the RADC Spectrum Estimation Workshop.

[7] R. ROY and T. KAILATH, "ESPRIT - Estimation of Signal Parameters via Rotational Invariance Techniques," IEEE Transactions On Acoustics, Speech, Signal Processing, vol. 37, no. 7, pp. 984-995, July 1989.

[8] A. PAULRAJ and T. KAILATH, "Eigenstructure methods for direction of arrival estimation in the presence of unknown noise field," IEEE Transactions On Aerospace and Electronic Systems, vol. 34, no. 1, pp. 13-20, February 1986.

[9] E. FERRARA and T. PARKS, "Direction finding with an array of antennas having diverse polarizations," IEEE Transactions On Antennas Propagation, vol. 31, pp. 231-236, March 1983.

[10] P. FORSTER and C. NIKIAS, "Bearing estimation in the bispectrum domain," IEEE Transactions On Signal Processing, vol. 39, no. 9, pp. 1994-2006, September 1991.

[11] S. SHAMSUNDER and G. B. GIANNAKIS, "Signal selective localization of nongaussian cyclostationary sources," IEEE Transactions on Speech and Audio Processing, vol. 42, no. 10, pp. 2860-2864, October 1994.

[12] P. CHEVAliER, A. FERREOL, L. ALBERA, and G. BIROT, "Higher order direction finding from arrays with diversely polarized antennas: The pd-2q-music algorithms," IEEE Transactions on Signal Processing, vol. 55, no. 11, pp. 5337-5350, November 2007.

[13] B. PORAT and B. FRIEDLANDER, "Direction finding algorithms based on high-order statistics," IEEE Transactions On Signal Processing, vol. 39, no. 9, pp. 2016-2024, September 1991.

[14] P. CHEVALIER, A. FERREOL, and L. ALBERA, "Highresolution direction finding from higher order statistics: The $2 q-$ MUSIC algorithm," IEEE Transactions on Signal Processing, vol. 54, no. 8, pp. 2986-2997, August 2006.

[15] I. ZISKIND and M. WAX, "Maximum likelihood localization of multiple sources by alternating projection," IEEE Transactions on Acoustic, Speech and Signal Processing, vol. 36, no. 10, pp. 1553-1560, October 1988.

[16] S. K. OH and C. K. UN, "A sequential estimation approach for performance improvement of eigenstructure-based methods in array processing," IEEE Transactions On Signal Processing, vol. 41, pp. 457-463, 1993.

[17] J. C. MOSHER and R. M. LEAHY, "Source localization using Recursively Applied and Projected (RAP) MUSIC," IEEE Transactions On Signal Processing, vol. 47, no. 2, pp. 332-340, February 1999. 
[18] P. STOICA, P. HANDEL, and A. NEHORAI, "Improved sequential music," IEEE Transactions On Aerospace and Electronic Systems, vol. 31, no. 4, pp. 1230-1239, October 1995.

[19] F. LI and R. VACCARO, "Sensitivity analysis of DOA estimation algorithms to sensor errors," IEEE Transactions On Aerospace and Electronic Systems, vol. 28, no. 3, pp. 708-717, July 1992.

[20] A. L. SWINDLEHURST and T. KAILATH, "A performance analysis of subspaced-based methods in the presence of model errors, part I: The MUSIC algorithm," IEEE Transactions On Signal Processing, vol. 40, no. 3, pp. 1758-1773, July 1992.

[21] P. McCULLAGH, Tensor Methods in Statistics. Chapman and Hall, Monographs on Statistics and Applied Probability, 1987.

[22] P. AMBLARD, M. GAETA, and J. LACOUME, "Statistics for complex variables and signals - parts I and II," Signal Processing, Elsevier, vol. 53, pp. 1-25, August 1996.

[23] L. ALBERA, A. FERREOL, P. COMON, and P. CHEVALIER, "Blind Identification of Overcomplete Mixtures of sources (BIOME)," Linear Algebra Applications, vol. 391C, pp. 3-30, November 2004.

[24] P. CHEVAlIER, L. ALBERA, A. FERREOL, and P. COMON, "On the virtual array concept for higher order array processing," IEEE Transactions On Signal Processing, vol. 53, no. 4, pp. 1254-1271, April 2005.

[25] A. FERREOL and P. CHEVALIER, "On the behavior of current second and higher order blind source separation methods for cyclostationary sources," IEEE Transactions On Signal Processing, vol. 48, no. 6, pp. 1712-1725, June 2000, Errata vol. 50, no. 4, pp. 990, April 2002.

[26] A. FERREOL, P. CHEVALIER, and L. ALBERA, "Second order blind separation of first and second order cyclostationary sources - Application to AM, FSK, CPFSK and deterministic sources," IEEE Transactions On Signal Processing, vol. 52, pp. 845-861, April 2004.

[27] C. D. MEYER, Matrix Analysis and Applied Linear Algebra. SIAM, 2000.

[28] H. W. SORENSON, Parameter Estimation : Principles and Problems. New York : Marcel Dekker, 1980.

[29] J. G. PROAKIS, Digital Communications, third edition. New York : McGrawHill, 1995.

[30] A. SMILDE and R. B. P. GELADI, Multi-way Analysis. John Wiley \& Sons Ltd, England, 2004.

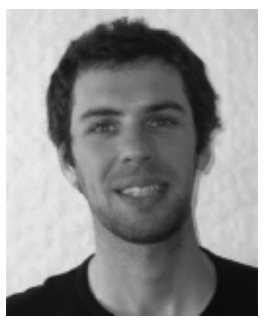

GwÃl'naÃńl Birot was born in La Roche-SurYon, France, in 1982. He received the Master 2 research degree in Signal, Teledetection, Radar, Antennas, Micro-waves, Propagation (TRAMP) and Image processing (STI), University of Rennes 1, Rennes, France, in 2006. He received in 2009 the Ph.D. degree in Signal Pocessing and Telecommunications from the University of Rennes 1 at the Laboratoire du Traitement du Signal et de lâÁźImage (LTSI). He is now with the National Instutute of Health and Medical Research (INSERM). His research interest includes higher order sources localization methods in both applications telecommunications and biomedical engineering. $\mathrm{He}$ also works on the forward problem in brain modeling.

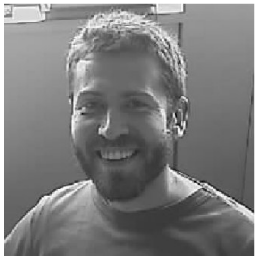

Laurent Albera (M'04) was born in Massy, France, in 1976. After a DESS in Mathematics, He received in 2001 the DEA's degree in Automatic and Signal Processing from the University of Science of Orsay (Paris XI). From 2001 to 2004, he was committed for a study within the framework of a Research Contract CIFRE (Convention Industrielle de Formation par la Recherche) between Thales Communications (formerly Thomson-CSF), the french National Centre for Scientific Research CNRS and the University of Nice SophiaAntipolis. He received in 2003 the Ph.D. degree in Sciences from the University of Nice Sophia-Antipolis, France. He is now Assistant Professor at the University of Rennes 1 and is affiliated with the INSERM research group LTSI (Laboratoire Traitement du Signal et de l'Image). His research interests include Multilinear Algebra and High-Order Statistics in order to perform Independent Component Analysis (ICA) and source localization.

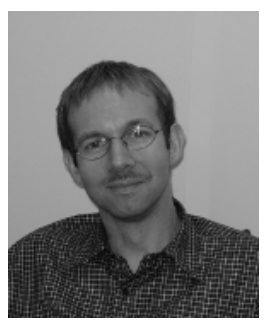

Pascal Chevalier received the M.Sc degree from Ecole Nationale SupÃl'rieure des Techniques AvancÃl'es (ENSTA), the Ph.D. degree from South-Paris University and the Habilitation Ãă Diriger des Recherches from Marne La VallÃl'e University, France, in 1985, 1991 and 2009 respectively. Since 1991 he has been with Thomson-CSF/RGS (now ThalÃĺsCommunications) where has shared industrial activities (studies, experimentations, expertises, management), teaching activities both in French engineer schools (ESE, ENST, ENSTA, ESIEE) and French Universities (Cergy-Pontoise) and research activities. Since 2000, he has also been acting as Technical Manager and Architect of the array processing sub-system as part of a national program of military satellite telecommunications. He is currently a ThalÃĺs Expert since 2003. His present research interests are in array processing techniques, either blind or informed, second order or higher order, Time-Invariant or Time-Varying especially for cyclostationary signals, linear or non linear and particularly widely linear for non circular signals, for applications such as TDMA and CDMA radiocommunications networks, satellite telecommunications, spectrum monitoring and passive listening in HVUHF band. Dr Chevalier has been a member of the THOMSON-CSF Technical and Scientifical Council between 1995 and 1998. He co-received the 2003 âĂIJScience and DefenseâĂİ Award from the french Ministry of Defence for its work as a whole about array processing for military radiocommunications. He is author or co-author of 26 patents and more than 120 papers (Journal, Conferences and Chapters of books). Dr. Chevalier is presently a member of the editorial board of the Eurasip Journal of Wireless Communications and Networking, an Associate Editor of the French Journal Traitement du Signal and an emeritus member of the SocietÃl' des Electriciens et des Electroniciens (SEE). 